\title{
Spectroscopy of large PAHs
}

\section{Laboratory studies and comparison to the Diffuse Interstellar Bands}

\author{
R. Ruiterkamp ${ }^{1}$, T. Halasinski ${ }^{2}$, F. Salama ${ }^{2}$, B. H. Foing ${ }^{3}$, L. J. Allamandola ${ }^{2}$, W. Schmidt ${ }^{4}$, and P. Ehrenfreund ${ }^{1}$ \\ 1 Leiden Observatory, PO Box 9513, 2300 RA Leiden, The Netherlands \\ 2 Space Science Division, NASA-Ames Research Center, Moffett Field CA 94035, USA \\ 3 ESA Research Support Division, ESTEC/SCI-SR, PO Box 229, 2200 AG, Noordwijk, The Netherlands \\ 4 PAH Forschungs Institut, Flurstrasse 17, 86926 Greifenberg/Ammersee, Germany
}

Received 16 January 2002 / Accepted 27 March 2002

\begin{abstract}
Polycyclic Aromatic Hydrocarbons (PAHs) are thought to be the carriers of the ubiquitous infrared emission bands (UIBs). Data from the Infrared Space Observatory (ISO) have provided new insights into the size distribution and the structure of interstellar PAH molecules pointing to a trend towards larger-size PAHs. The mid-infrared spectra of galactic and extragalactic sources have also indicated the presence of 5-ring structures and PAH structures with attached side groups. This paper reports for the first time the laboratory measurement of the UV-Vis-NIR absorption spectra of a representative set of large PAHs that have also been selected for a long duration exposure experiment on the International Space Station ISS. PAHs with sizes up to $600 \mathrm{amu}$, including 5-ring species and PAHs containing heteroatoms, have been synthesized and their spectra measured using matrix isolation spectroscopy. The spectra of the neutral species and the associated cations and anions measured in this work are also compared to astronomical spectra of Diffuse Interstellar Bands (DIBs).
\end{abstract}

Key words. molecular data - methods: laboratory - ISM: lines and bands - ISM: molecules

\section{Introduction}

A substantial fraction of the ca. 120 gas phase molecules that have been identified in interstellar and circumstellar regions are organic in nature (Ehrenfreund \& Charnley 2000). Most of these molecules have been detected through their rotational lines. Large carbon-bearing molecules (such as polycyclic aromatic hydrocarbons (PAHs), fullerenes and unsaturated chains) are also thought to be present in the interstellar medium (ISM). These large molecules are difficult to identify in the radio range and can only be detected through their electronic or vibrational signature in the UV-Vis-IR range.

The presence of large aromatic structures is evidenced by infrared observations of the ISM in our galaxy and in extragalactic environments (see A\&A Vol. 315, 1996) and by the observation of the Diffuse Interstellar Bands (DIBs) in the visible range (see Herbig 1995 for a review, Tielens \& Snow 1995). Hydrogenated amorphous carbon (HAC) is considered a potential carrier for the $2200 \AA$ bump observed in the interstellar extinction curve (Mennella et al. 1999), while a variety of complex aromatic networks are likely to be present on carbonaceous grains (see Henning \& Salama 1998 for a review). Since the initial discovery of simple diatomic molecules in interstellar space (Swings \& Rosenfeld 1937; McKellar 1940;

Send offprint requests to: R. Ruiterkamp, e-mail: ruiterka@strw.leidenuniv.nl
Douglas \& Herzberg 1941); many more molecules have been detected in the diffuse medium, such as $\mathrm{HCO}^{+}, \mathrm{CO}, \mathrm{OH}, \mathrm{C}_{2}$, $\mathrm{HCN}, \mathrm{CN}, \mathrm{CS}$ and $\mathrm{H}_{2} \mathrm{CO}$ (Lucas \& Liszt 1997). Models of gasphase reactions in diffuse clouds predict that organic molecules can be formed through ion-molecule reactions and neutralneutral reactions in the diffuse medium (Bettens \& Herbst 1996). Aromatic and unsaturated linear carbon molecules of up to $64 \mathrm{C}$ atoms have been predicted by these models (Herbst 1995; Ruffle et al. 1999). Reactions in circumstellar envelopes and subsequent mixing into the diffuse medium, photochemical reactions and grain collisions in carbonaceous dust are alternative processes that can also lead to the formation of organic molecules.

Here, we report the spectroscopy of large PAHs (see Fig. 1). Although extensive laboratory studies have been performed on the spectroscopy of smaller PAHs (i.e., $<25 \mathrm{C}$-atoms molecules) in an astrophysical context, almost no information is available regarding large PAHs ( $>25 \mathrm{C}$ atoms, for a recent review see Salama 1999).

The PAH molecules shown in Fig. 1 have also been selected for a long duration exposure experiment "Organic Matter in Space" on the International Space Station (ISS) (Ruiterkamp et al. 2001). This space experiment will provide quantitative data on the photochemical evolution of specific PAH species in irradiated environments. The results will help to constrain the formation and destruction pathways of large carbonaceous 


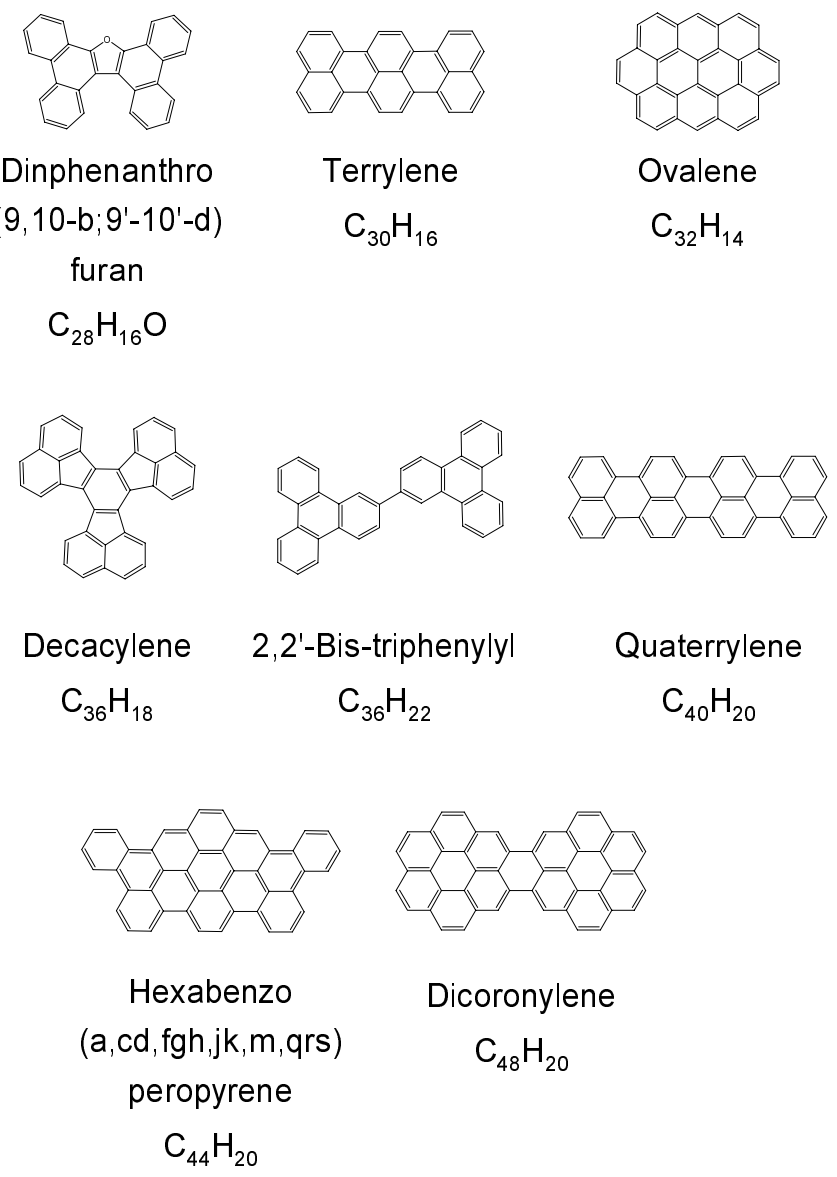

Fig. 1. Structural formulae of the Polycyclic Aromatic Hydrocarbon molecules studied by UV-Vis-NIR spectroscopy.

molecules in interstellar and interplanetary media. The sample set includes regular compact and non-compact PAHs (i.e., structures made only of benzenoid rings), PAH structures with 5-membered rings as well as one oxygen-bearing PAH.

Thus, the objectives of this work are: (i) to explore for the first time the electronic spectroscopy of large PAH molecules and ions in an environment that is astrophysically relevant for comparison with the spectra of DIBs; (ii) to identify promising target molecules for further studies in gas-phase, jet expansion, experiments; (iii) to study the photo-stability of these selected species in the laboratory for a comparison with samples to be exposed on the ISS.

In Sect. 2 we briefly review the evidence pointing toward the presence of PAHs in the ISM and the potential connection to the DIBs. In Sect. 3 we describe the laboratory setup used in this study. In Sect. 4 we report and discuss the UV-Vis-NIR absorption spectra of selected large, neutral and ionized, PAH molecules in the astrophysical context. The spectra are finally compared with high resolution DIB data in Sect. 5.

\section{The carrier molecules of the diffuse interstellar bands}

\subsection{The Diffuse Interstellar Bands}

Diffuse Interstellar Bands (DIBs) are absorption lines that are detected in the spectra of reddened stars throughout our galaxy.
They are observed in a spectral range extending from the UV to the near-IR, and they exhibit a large diversity of band strengths and profiles (Herbig 1995). The detection of DIBs in almost all regions of the diffuse ISM indicates that the carriers are chemically stable.

High resolution surveys of the DIBs indicate that the band strengths depend on the environmental conditions that prevail in the ISM (Herbig 1975; Cami et al. 1997; Sonnentrucker et al. 1997). The DIB carriers are strongly influenced by the UV field. The shielding of UV radiation by the outer layers of the clouds leads to a progressive reduction of DIB strength towards the interior of the cloud (Cami et al. 1997).

The current consensus based on both astronomical observations and laboratory studies is that the DIB carriers are gas phase molecules that are chemically stable and carbonaceous in nature (Herbig 1995; Tielens \& Snow 1995; Henning \& Salama 1998; Ehrenfreund \& Charnley 2000; Ehrenfreund et al. 2001). The observation of some DIBs in emission in the Red Rectangle (Sarre et al. 1995) and the detection of intrinsic substructures in several of the stronger DIBs marked a breakthrough in the identification of the molecular nature of the DIB carriers (Sarre et al. 1995; Ehrenfreund \& Foing 1996; Krelowski et al. 1998; Le Coupanec et al. 1999; Walker et al. 2001).

\subsection{Proposed carbonaceous carriers}

Among the potential DIB carriers are stable carbonaceous molecules such as PAHs, fullerenes, carbon chains and rings. In order to identify the carrier molecules of DIBs, laboratory studies have been carried out on PAHs (Salama et al. 1996; Salama et al. 1999) and carbon chains (Freivogel et al. 1994; Tulej et al. 1998). There is, however, no definitive identification of one specific carrier for any of the 300 known DIBs. It seems logical to seek the carriers of the DIBs among interstellar molecules that are both stable and cosmically abundant. In this report we focus on PAHs. The spectroscopy of large fullerenes will be discussed in a separate paper. PAHs are believed to be the most abundant free organic molecules in space (Puget \& Leger 1989; Allamandola et al. 1989) and to be formed in the outer atmosphere of carbon stars, or by shock fragmentation of carbonaceous solid material. The infrared emission bands (UIBs) measured at 3.3, 6.2, 7.7, 8.6, 11.2 and $12.7 \mu \mathrm{m}$ are ubiquitous in space. The bands are observed in the diffuse interstellar medium, in circumstellar environments and even in external galaxies (Tielens et al. 1999). UIBs have been successfully modeled by combining the infrared laboratory absorption spectra of neutral and positively charged PAHs (Hudgins \& Allamandola 1999a, b).

PAHs play an important role in the evolution of interstellar gas phase chemistry (Bakes \& Tielens 1994). Gaseous PAH molecules react according to the environmental conditions. Neutral and hydrogenated PAHs are present in dense regions and regions of high electron density. The local ultraviolet radiation field determines their charge and their hydrogenation states (Salama et al. 1996; Sonnentrucker et al. 1997; Vuong \& Foing 2000). Furthermore, the UV-Vis spectra of PAH cations 
with up to $100 \mathrm{C}$ atoms show strong absorption bands. This makes PAHs promising candidates for the molecular carriers of the diffuse interstellar bands. The search for specific bands of PAH cations in highly reddened stars has shown some similarities with the spectra of small PAHs measured in the laboratory in neon matrices but no definitive identification can be made until astrophysically relevant gas-phase data are available. Although PAHs appear to be potential carrier molecules, other species have to be explored, e.g. fullerenes such as the cation $\mathrm{C}_{60}^{+}$have been discussed (Foing \& Ehrenfreund 1994; Foing \& Ehrenfreund 1997). Laboratory spectroscopy experiments provide a vital tool for the identification of the DIB carriers in the ISM.

\section{Experimental}

The molecules used in this study were synthesized via condensation processes of small units (e.g. naphthalene) according to well established literature methods (Clar 1964). The laboratory spectra were obtained at NASA-Ames using the techniques of Matrix Isolation Spectroscopy (MIS). This technique approximates the known (or expected) environmental conditions of the diffuse interstellar medium in the laboratory. In MIS experiments, neutral and ionized PAHs are fully isolated in lowpolarizability neon matrices at $<5 \mathrm{~K}$. The induced perturbations - in terms of peak position and profile of the bands - in the spectra of the neutral/ionized PAHs are minimal (Salama 1996). The experimental apparatus and protocol have been described previously (Salama et al. 1999), and here we only provide a brief description. A sample holder is suspended in the center of a sample chamber that is part of a high vacuum system. The sample chamber consists of four ports at $90^{\circ}$ and a gas injection port at $45^{\circ}$. The sample holder is cooled to $4.2 \mathrm{~K}$ using a liquid Helium transfer cryostat. The substrate can be rotated to face a vacuum deposition furnace, the spectroscopy ports, a VUV irradiation lamp and the inert gas injection port, respectively. Spectra of the isolated $\mathrm{PAH}$ molecules were obtained with a $0.5-\mathrm{m}$, triple grating monochromator and a CCD array mounted on the exit port, and interfaced to a computer system. The spectral light sources consist of a $D_{2}$ lamp (UV) and a tungsten filament lamp (Vis - NIR). The light collected at the spectroscopy port of the sample chamber is directed to the entrance slit of the monochromator by a fiber optic cable. The wavelength coverage of the spectrometer system is 180$1200 \mathrm{~nm}$. Wavelength calibration of the spectrometer is provided by the emission lines of a neon and a Mercury lamp. The mean of the deviation compared to literature values was used to calibrate the entire spectrum. Calibration shifts did not exceed $0.05 \mathrm{~nm}$.

A microwave-powered, flow-discharge hydrogen lamp generating $10.2 \mathrm{eV}$ photons $\left(L_{\alpha}\right)$ is used for irradiation. Note that in the case of large molecules with low ionization potential (IP), the Deuterium lamp also can ionize the sample before irradiation with $L_{\alpha}$. In typical experiments, PAH samples are simultaneously condensed with the neon gas onto the cold substrate, and the frozen matrix is then spectroscopically probed. The matrix is then exposed to VUV irradiation leading to the formation of ions from the neutral precursor and the observation of new spectral features in the UV-NIR range (180-1200 nm). The new features are associated with the formation of ions in the matrix through one-photon ionization of the neutral PAH precursor. All samples were irradiated for 10 min leading to the formation of cation and anion pairs in the neon matrix. Additional experiments were performed in each case to discriminate between the spectral signatures of the ion and its counter ion. This was done by doping the matrix with an electron scavenger, $\mathrm{NO}_{2}$, to ensure that only cations were formed in the matrix (Jacox 1978). Comparison between the two sets of experiments allows an unambiguous determination of the signature associated with each ion and its counter ion in the matrix. The spectra of matrices doped with $\mathrm{NO}_{2}$ exhibit $\mathrm{NO}_{2}$ bands that peak at $570 \mathrm{~nm}$, $604 \mathrm{~nm}$ and $643 \mathrm{~nm}$. These features have been omitted from the tables in the results section. Although all features above the 0.02 absorption level have been taken into account, a few broad features falling above $1100 \mathrm{~nm}$ with residuals at the $0.02 \mathrm{ab}-$ sorbance level (probably due to matrix effects) have been omitted from the discussion.

\section{Results and discussion}

Figures 2 to 9 show the absorption spectra of neutral and ionized PAHs. Note that for each figure, the left panel shows the absorption spectrum of the matrix-isolated neutral PAH while the right panel shows the spectrum derived from the difference between the spectra of the same PAH measured before and after irradiation of the matrix respectively.

The "difference spectrum" is defined as the negative logarithm of the single beam intensity associated with the ionized species divided by the single beam intensity associated with the neutral species. The difference spectra show a negative absorbance for the neutral precursor that is depleted into ions and a positive absorbance for the ions that are produced. The tables list the peak positions, Full Width at Half Maximum (FWHM) and peak heights of the bands measured in the laboratory.

\subsection{Neutral and ionized diphenanthrofuran $\mathrm{C}_{28} \mathrm{H}_{16} \mathrm{O}$}

The absorption spectrum of neutral diphenanthrofuran is shown in Fig. 2. The spectrum is characterized by a broad absorption band system peaking at $262.5 \mathrm{~nm}$ and two band systems with pronounced fine structure seen around $331.0 \mathrm{~nm}$ and $360 \mathrm{~nm}$, respectively. Upon photolysis, seven new additional features appear in the spectrum. The new features include a band system comprised of three peaks centered near $426.0 \mathrm{~nm}$ and a multiple peak structure centered around $618.4 \mathrm{~nm}$. Comparison of this spectrum with the results of a $\mathrm{Ne} / \mathrm{NO}_{2}$ experiment shows that a peak seen at 752.8 after VUV irradiation is absent when the Ne matrix is doped with $\mathrm{NO}_{2}$. The $752.8 \mathrm{~nm}$ band can therefore be confidently assigned to the anion of diphenanthrofuran $\mathrm{C}_{28} \mathrm{H}_{16} \mathrm{O}^{-}$. The peak position, intensity and $F W H M$ for each band are given in Table 1.

\subsection{Neutral and ionized terrylene $\mathrm{C}_{30} \mathrm{H}_{16}$}

The spectrum of neutral terrylene (Fig. 3) shows a broad band system around $220 \mathrm{~nm}$ and a narrower, strong, absorption band system with substructure in the visible $(450-600 \mathrm{~nm}$ 

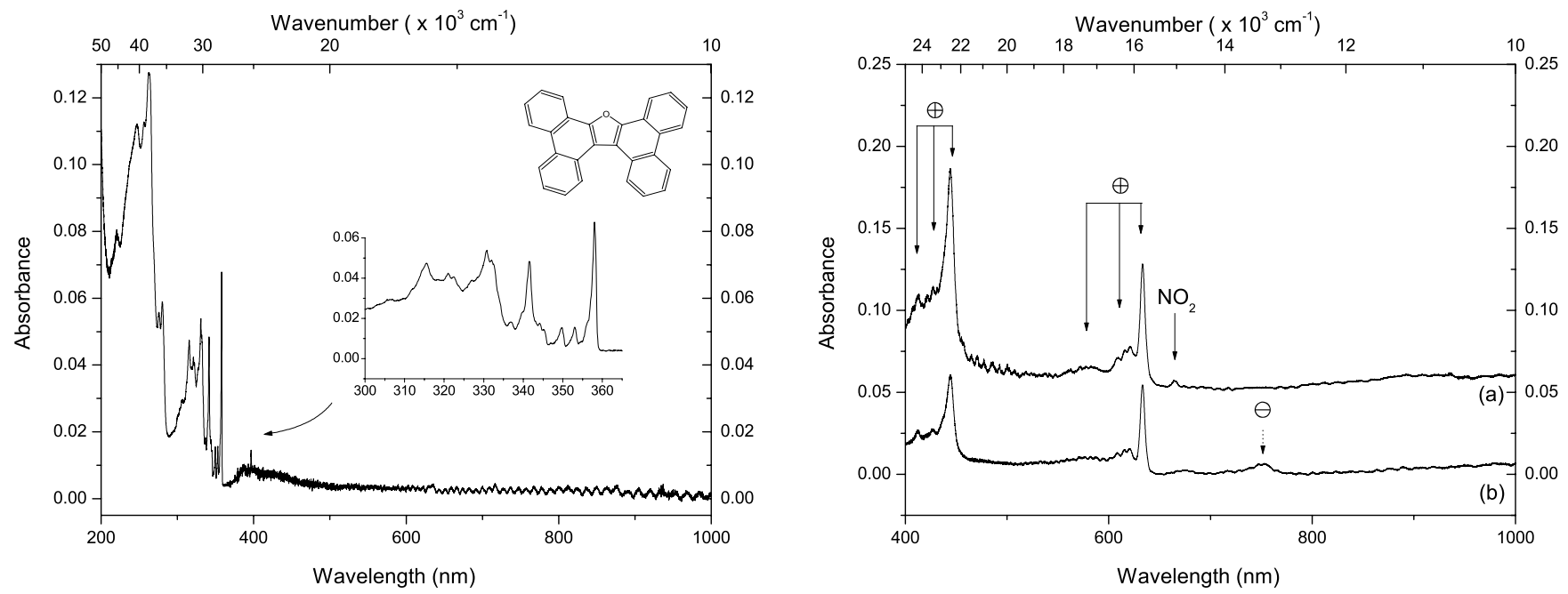

Fig. 2. Comparison of the spectral features associated with neutral (left panel) and VUV-irradiated (right panel) diphenanthrofuran (Dp) isolated in neon $(\mathrm{Ne})$ and in $\mathrm{Ne} / \mathrm{NO}_{2}$ matrices. a) is the spectrum of the photolysis products in a neon/ $\mathrm{NO}_{2}$ matrix; $\mathbf{b}$ ) is the spectrum of the photolysis products, including $\mathrm{Dp}^{+}$and $\mathrm{Dp}^{-}$, in a neon matrix. Band peak positions, intensities and $F W H M$ are reported in Table 1.

Table 1. Absorption features associated with the UV/Vis/NIR spectra of neutral and ionized diphenanthrofuran $\left(\mathrm{C}_{28} \mathrm{H}_{16} \mathrm{O}\right)$ isolated at $4 \mathrm{~K}$ in inert-gas matrices.

Neutral in neon matrix

10 min. irr. in neon matrix

10 min. irr. in neon/ $\mathrm{NO}_{2}$ matrix

\begin{tabular}{lcccccccc}
\hline \hline $\begin{array}{l}\text { Peak pos. } \\
(\mathrm{nm})\end{array}$ & $\begin{array}{c}F W H M \\
(\mathrm{~nm})\end{array}$ & $A b s_{\max }$ & $\begin{array}{c}\text { Peak pos. } \\
(\mathrm{nm})\end{array}$ & $\begin{array}{c}F W H M \\
(\mathrm{~nm})\end{array}$ & $A b s_{\max }$ & $\begin{array}{c}F W H M \\
(\mathrm{~nm})\end{array}$ & $A b s_{\max }$ & Assignment \\
\hline $220.95(\mathrm{~B})$ & 6.6 & 0.099 & 413.37 & 4.9 & 0.009 & 6.8 & 0.007 & + \\
$246.67(\mathrm{~B})$ & 6.2 & 0.112 & 425.99 & 4.3 & 0.027 & 2.9 & 0.064 & + \\
$256.46(\mathrm{~B})$ & 3.9 & 0.113 & 444.43 & 10.3 & 0.055 & 7.4 & 0.134 & + \\
$262.51(\mathrm{~B})$ & 7.9 & 0.130 & 578.26 & 43.7 & 0.004 & 37.3 & 0.006 & + \\
$275.79(\mathrm{~B})$ & 2.4 & 0.056 & 617.36 & 11.4 & 0.015 & 6.1 & 0.026 & + \\
$280.25(\mathrm{~B})$ & 4.5 & 0.059 & 633.17 & 4.9 & 0.053 & 5.5 & 0.075 & + \\
$315.40(\mathrm{~B})$ & 1.7 & 0.047 & 752.82 & 19.5 & 0.006 & & & \\
$321.01(\mathrm{~B})$ & 1.0 & 0.042 & & & & & & \\
$331.04(\mathrm{~B})$ & 5.3 & 0.052 & & & & & & \\
$336.75(\mathrm{~B})$ & 2.1 & 0.018 & & & & & & \\
341.46 (B) & 1.1 & 0.048 & & & & & & \\
349.57 & 1.0 & 0.015 & & & & & & \\
352.94 & 0.9 & 0.015 & & & & & & \\
357.92 & 0.9 & 0.066 & & & & & \\
1073.70 & 3.6 & 0.010 & & & & & & \\
\hline
\end{tabular}

(B) $=$ Blended peak. Assignments only apply to the peaks that are detected in the spectra of the irradiated sample. $+=$ cation; $-=$ anion.

range). VUV irradiated terrylene exhibits new absorptions including two strong bands at $690.4 \mathrm{~nm}$ and $741.3 \mathrm{~nm}$. A close comparison of the spectra with the spectrum of a $\mathrm{Ne} / \mathrm{NO}_{2}$ doped matrix shows that the $741.3 \mathrm{~nm}$ band belongs to the anion as well as a few other, weaker, features. The values for the peak positions, intensities and FWHM are given in Table 2.

\subsection{Neutral and ionized ovalene $\mathrm{C}_{32} \mathrm{H}_{14}$}

Three major absorption band systems are seen in the spectrum of neutral ovalene shown in Fig. 4. A broad absorption peaking around $215.8 \mathrm{~nm}$, a band system falling in the $270-340 \mathrm{~nm}$ range and another, narrower, band system falling between 340 and $430 \mathrm{~nm}$. This spectrum is in agreement with the information available in the literature (Ehrenfreund et al. 1992). After exposure to VUV irradiation, the main new features that arise in the spectrum peak at 463.2, 535.7, 562.1 and $974.9 \mathrm{~nm}$. Band peak positions, intensities and $F W H M$ are reported in Table 3.

\subsection{Neutral and ionized decacyclene $\mathrm{C}_{36} \mathrm{H}_{18}$}

The spectrum of neutral decacyclene isolated in a neon matrix is shown in Fig. 5. The spectrum is composed of two broad absorption band systems centered around 251.8 and $364.2 \mathrm{~nm}$, respectively with superimposed fine structure. New broad bands 

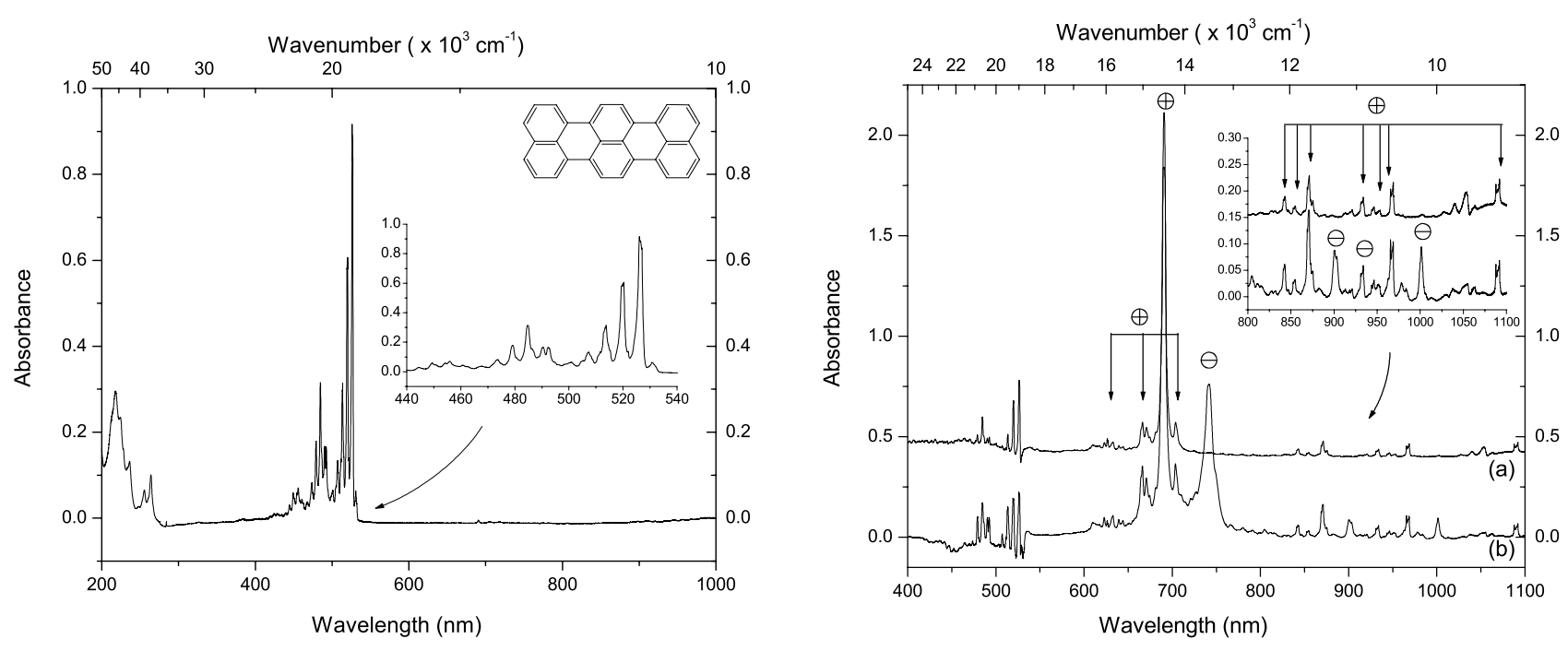

Fig. 3. Comparison of the spectral features associated with neutral (left panel) and VUV-irradiated (right panel) terrylene (Te) isolated in neon $(\mathrm{Ne})$ and in $\mathrm{Ne} / \mathrm{NO}_{2}$ matrices. a) is the spectrum of the photolysis products in a neon/ $/ \mathrm{NO}_{2}$ matrix; $\mathbf{b}$ ) is the spectrum of the photolysis products, including $\mathrm{Te}^{+}$and $\mathrm{Te}^{-}$, in a neon matrix. Band peak positions, intensities and $F W H M$ are reported in Table 2.
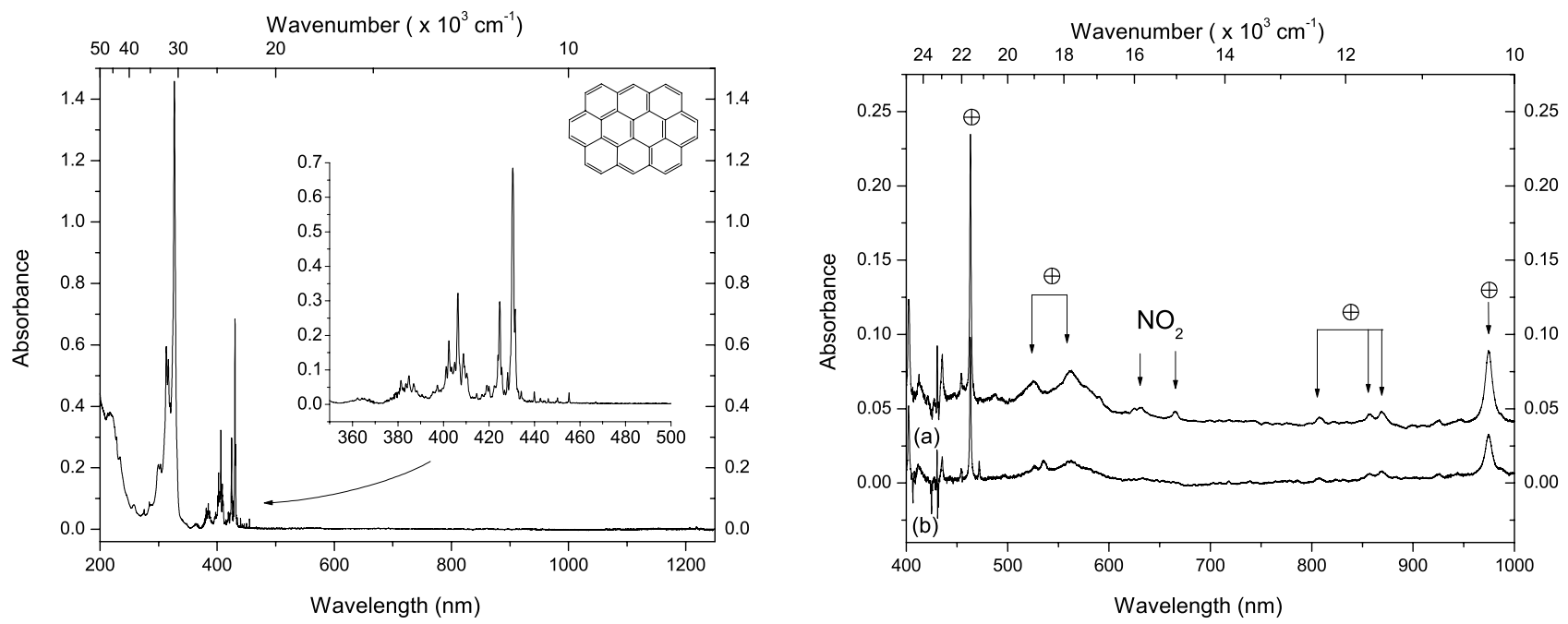

Fig. 4. Comparison of the spectral features associated with neutral (left panel) and VUV-irradiated (right panel) ovalene (Ov) isolated in neon $(\mathrm{Ne})$ and in $\mathrm{Ne} / \mathrm{NO}_{2}$ matrices. a) is the spectrum of the photolysis products in a neon/ $\mathrm{NO}_{2}$ matrix; $\mathbf{b}$ ) is the spectrum of the photolysis products in a neon matrix. Band peak positions, intensities and $F W H M$ are reported in Table 3.

appear at 539.7, 813.0 and 1095.8 after VUV irradiation of decacyclene in a $\mathrm{Ne} / \mathrm{NO}_{2}$ matrix. All the bands are assigned to the decacyclene cation $\left(\mathrm{C}_{36} \mathrm{H}_{18}^{+}\right)$. Band peak positions, intensities and FWHM are reported in Table 4.

\subsection{Neutral and ionized 2, 2' -bis-triphenylyl $\mathrm{C}_{36} \mathrm{H}_{22}$}

The spectrum of neutral triphenylyl (Fig. 6) shows strong absorption bands in the $200-350 \mathrm{~nm}$ range. In the case of the VUV-irradiated sample, both triphenylyl/Ne and triphenylyl $/ \mathrm{Ne} / \mathrm{NO}_{2}$ experiments exhibit two strong bands near 457.7 and $490.4 \mathrm{~nm}$, respectively, associated with the formation of the cation $\left(\mathrm{C}_{36} \mathrm{H}_{22}^{+}\right)$in the matrix. Band peak positions, intensities and $F W H M$ are reported in Table 5.

\subsection{Neutral and ionized quaterrylene $\mathrm{C}_{40} \mathrm{H}_{20}$}

The spectrum of neutral quaterrylene (Fig. 7) exhibits a complex band system between 200 and $320 \mathrm{~nm}$. A strong absorption band system is also found in the visible range between 450 and $660 \mathrm{~nm}$ with a peak at $607.9 \mathrm{~nm}$. VUV irradiation of quaterrylene leads to the appearance of two new, strong, absorption bands located at $833.7 \mathrm{~nm}$ and $881.8 \mathrm{~nm}$, respectively, together with weaker features down to the $1100 \mathrm{~nm}$ range. Comparison with the spectrum of a $\mathrm{Ne} / \mathrm{NO}_{2}$ matrix experiment indicates that the strong $881.8 \mathrm{~nm}$ band is associated with the absorption of the quaterrylene anion. Band peak positions, intensities and $F W H M$ are reported in Table 6.

Note that the spectrum of neutral quaterrylene (Fig. 7) resembles closely that of neutral terrylene (Fig. 3), the only difference being the shift in band positions. The bands of 
Table 2. Absorption features associated with the UV/Vis/NIR spectra of neutral and ionized terrylene $\left(\mathrm{C}_{30} \mathrm{H}_{16}\right)$ isolated at $4 \mathrm{~K}$ in inert-gas matrices.

Neutral in neon matrix 10 min. irr. in neon matrix 10 min. irr. in neon/ $\mathrm{NO}_{2}$ matrix

\begin{tabular}{lcccccccc}
\hline \hline $\begin{array}{l}\text { Peak pos. } \\
(\mathrm{nm})\end{array}$ & $\begin{array}{c}F W H M \\
(\mathrm{~nm})\end{array}$ & $A b s_{\max }$ & $\begin{array}{c}\text { Peak pos. } \\
(\mathrm{nm})\end{array}$ & $\begin{array}{c}F W H M \\
(\mathrm{~nm})\end{array}$ & $A b s_{\max }$ & $\begin{array}{c}F W H M \\
(\mathrm{~nm})\end{array}$ & $A b s_{\max }$ & Assignment \\
\hline $217.68(\mathrm{~B})$ & 11.6 & 0.298 & 609.96 & 3.0 & 0.074 & 5.8 & 0.049 & + \\
$223.61(\mathrm{~B})$ & 5.9 & 0.249 & 622.87 & 1.5 & 0.098 & 1.9 & 0.060 & + \\
$235.06(\mathrm{~B})$ & 6.7 & 0.145 & 626.20 & 0.9 & 0.032 & 0.9 & 0.041 & + \\
$255.33(\mathrm{~B})$ & 2.5 & 0.079 & 627.13 & 0.3 & 0.015 & 0.4 & 0.027 & + \\
$263.66(\mathrm{~B})$ & 3.3 & 0.108 & 632.16 & 2.6 & 0.107 & 3.7 & 0.062 & + \\
428.82 & 17.4 & 0.022 & 639.43 & 1.7 & 0.088 & 1.7 & 0.046 & + \\
439.74 & 3.1 & 0.025 & 643.90 & 1.9 & 0.082 & 2.6 & 0.04 & + \\
444.77 & 2.5 & 0.042 & 664.62 & 3.7 & 0.123 & 4.9 & 0.277 & + \\
449.88 & 3.2 & 0.068 & 670.68 & 2.2 & 0.293 & 2.2 & 0.136 & + \\
455.86 & 0.9 & 0.085 & 674.23 & 2.1 & 0.208 & 2.0 & 0.089 & + \\
460.80 & 3.6 & 0.054 & 690.39 & 4.4 & 1.908 & 3.4 & 1.712 & + \\
467.77 & 3.2 & 0.051 & 703.90 & 2.8 & 0.358 & 3.6 & 0.156 & + \\
473.54 & 2.6 & 0.092 & 741.32 & 10.9 & 0.722 & & & + \\
479.14 & 1.4 & 0.193 & 842.28 & 3.2 & 0.060 & 2.8 & 0.029 & + \\
484.78 & 1.5 & 0.328 & 854.05 & 3.3 & 0.031 & 3.1 & 0.010 & + \\
490.36 & 1.2 & 0.182 & 870.39 & 3.2 & 0.162 & 3.8 & 0.063 & + \\
492.41 & 1.5 & 0.181 & 874.97 & 1.9 & 0.049 & 2.1 & 0.029 & + \\
500.28 & 3.2 & 0.075 & 901.48 & 5.9 & 0.086 & & & + \\
507.27 & 1.9 & 0.144 & 932.65 & 3.4 & 0.051 & 3.5 & 0.023 & + \\
513.47 & 1.7 & 0.314 & 946.11 & 1.4 & 0.030 & 5.3 & 0.015 & + \\
519.79 & 1.5 & 0.637 & 966.74 & 4.1 & 0.091 & 1.0 & 0.047 & + \\
526.32 & 1.6 & 0.951 & 978.42 & 3.8 & 0.024 & & & + \\
530.83 & 2.8 & 0.072 & 983.29 & 4.4 & 0.014 & & & + \\
& & & 1001.20 & 3.5 & 0.088 & & & + \\
\hline
\end{tabular}

(B) $=$ Blended peak. Assignments only apply to the peaks that are detected in the spectra of the irradiated sample. $+=$ cation. $-=$ anion.
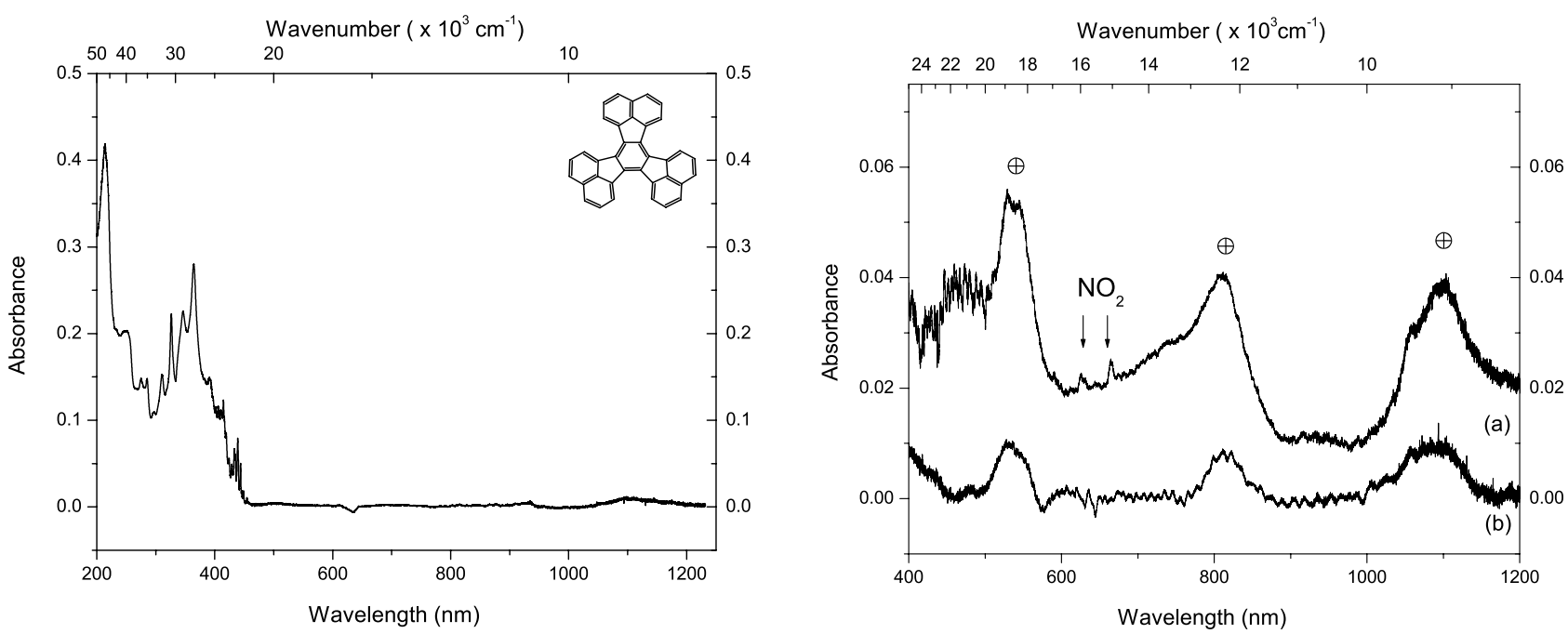

Fig. 5. Comparison of the spectral features associated with neutral (left panel) and VUV-irradiated (right panel) decacyclene (Dc) isolated in neon $(\mathrm{Ne})$ and in $\mathrm{Ne} / \mathrm{NO}_{2}$ matrices. a) is the spectrum of the photolysis products in a neon/ $\mathrm{NO}_{2}$ matrix; b) is the spectrum of the photolysis products in a neon matrix. Band peak positions, intensities and $F W H M$ are reported in Table 4.

quaterrylene are red-shifted compared to the bands of terrylene. The same effect is observed when comparing the spectra of their respective ions. This molecular size-related effect is well known and has been discussed in the literature (e.g. Clar 1964; Salama et al. 1996).

\subsection{Neutral and ionized hexabenzoperopyrene $\mathrm{C}_{44} \mathrm{H}_{20}$}

The spectrum of neutral hexabenzoperopyrene (Fig. 8) shows three band systems peaking at 236.0, 345.9, and $400.0 \mathrm{~nm}$, 
Table 3. Absorption features associated with the UV/Vis/NIR spectra of neutral and ionized ovalene $\left(\mathrm{C}_{32} \mathrm{H}_{14}\right)$ isolated at $4 \mathrm{~K}$ in inert-gas matrices.

\begin{tabular}{|c|c|c|c|c|c|c|c|c|}
\hline \multicolumn{3}{|c|}{ Neutral in neon matrix } & \multicolumn{3}{|c|}{10 min. irr. in neon matrix } & \multicolumn{3}{|c|}{10 min. irr. in neon/ $\mathrm{NO}_{2}$ matrix } \\
\hline $\begin{array}{l}\text { Peak pos. } \\
(\mathrm{nm})\end{array}$ & $\begin{array}{c}F W H M \\
(\mathrm{~nm})\end{array}$ & $A b s_{\max }$ & $\begin{array}{l}\text { Peak pos. } \\
\quad(\mathrm{nm})\end{array}$ & $\begin{array}{c}F W H M \\
(\mathrm{~nm})\end{array}$ & $A b s_{\max }$ & $\begin{array}{c}F W H M \\
(\mathrm{~nm})\end{array}$ & $A b s_{\max }$ & Assignment \\
\hline 215.81 (B) & 31.1 & 0.378 & 454.26 & 1.2 & 0.010 & 1.4 & 0.013 & + \\
\hline $257.46(\mathrm{~B})$ & 6.4 & 0.080 & 463.23 & 1.3 & 0.097 & 1.5 & 0.182 & + \\
\hline 275.21 & 1.1 & 0.063 & 471.91 & 0.9 & 0.010 & & & - \\
\hline 284.18 & 0.3 & 0.088 & 526.04 & 6.4 & 0.004 & 10.2 & 0.008 & + \\
\hline $299.36(\mathrm{~B})$ & 5.1 & 0.209 & 535.71 & 4.5 & 0.015 & & & - \\
\hline $303.83(\mathrm{~B})$ & 2.8 & 0.180 & 562.08 & 41.7 & 0.013 & 38.2 & 0.027 & + \\
\hline 313.17 (B) & 2.9 & 0.566 & 808.24 & 9.4 & 0.003 & 6.4 & 0.004 & + \\
\hline 316.81 (B) & 2.3 & 0.532 & 857.44 & 8.3 & 0.006 & 6.7 & 0.006 & + \\
\hline 319.34 (B) & 2.6 & 0.410 & 869.56 & 8.7 & 0.008 & 6.7 & 0.008 & + \\
\hline 326.97 & 3.8 & 1.341 & 974.92 & 7.2 & 0.031 & 8 & 0.046 & + \\
\hline 384.34 & 8.1 & 0.057 & 1074.75 & 11.7 & 0.011 & & & - \\
\hline 395.60 & 1.4 & 0.036 & 1125.45 & 48.8 & 0.015 & 26.1 & 0.03 & + \\
\hline 397.34 & 0.5 & 0.057 & & & & & & \\
\hline 402.41 & 0.4 & 0.184 & & & & & & \\
\hline 406.33 & 0.6 & 0.326 & & & & & & \\
\hline 408.85 & 1.2 & 0.137 & & & & & & \\
\hline 414.60 & 0.5 & 0.031 & & & & & & \\
\hline 417.05 & 0.9 & 0.028 & & & & & & \\
\hline 419.08 & 0.7 & 0.057 & & & & & & \\
\hline 420.08 & 0.5 & 0.050 & & & & & & \\
\hline 422.55 & 1.2 & 0.054 & & & & & & \\
\hline 423.99 & 0.4 & 0.141 & & & & & & \\
\hline 424.72 & 0.6 & 0.307 & & & & & & \\
\hline 425.62 & 0.5 & 0.105 & & & & & & \\
\hline 426.90 & 0.4 & 0.041 & & & & & & \\
\hline 428.20 & 0.5 & 0.092 & & & & & & \\
\hline 430.48 & 0.9 & 0.701 & & & & & & \\
\hline 431.47 & 0.6 & 0.267 & & & & & & \\
\hline 434.17 & 0.3 & 0.038 & & & & & & \\
\hline 439.94 & 0.3 & 0.036 & & & & & & \\
\hline 442.58 & 0.4 & 0.018 & & & & & & \\
\hline 444.29 & 0.9 & 0.010 & & & & & & \\
\hline 446.04 & 0.3 & 0.017 & & & & & & \\
\hline 450.11 & 0.3 & 0.019 & & & & & & \\
\hline 451.52 & 0.5 & 0.007 & & & & & & \\
\hline 455.19 & 0.3 & 0.034 & & & & & & \\
\hline
\end{tabular}

(B) $=$ Blended peak. Assignments only apply to the peaks that are detected in the spectra of the irradiated sample. $+=$ cation; $-=$ anion.

respectively. VUV photolysis leads to the formation of three new absorption features that appear in the spectrum at $525.6 \mathrm{~nm}, 562.5$ and $574.8 \mathrm{~nm}$ respectively. $\mathrm{A} \mathrm{Ne} / \mathrm{NO}_{2}$ matrix experiment clearly indicates that the band at $574.8 \mathrm{~nm}$ can be assigned to absorption by the anion $\left(\mathrm{C}_{44} \mathrm{H}_{20}^{-}\right)$. Band peak positions, intensities and $F W H M$ are reported in Table 7.

\subsection{Neutral and ionized dicoronylene $\mathrm{C}_{48} \mathrm{H}_{20}$}

The absorption spectra of neutral and ionized dicoronylene have recently been studied in detail (Chen et al. 2001). The results are briefly reviewed here. The spectrum of neutral dicoronylene (Fig. 9) isolated in a neon matrix exhibits three structured band systems around 248.3, 333.3 and $444.6 \mathrm{~nm}$. These band systems are all depleted upon irradiation of the neutral dicoronylene. Seven new peaks appear in the spectrum upon photolysis. The spectrum of the ion is clearly dominated by two strong bands peaking at $682.5 \mathrm{~nm}$ and $1017.1 \mathrm{~nm}$. Comparison with a $\mathrm{Ne} / \mathrm{NO}_{2}$ matrix indicates that all the new peaks can be assigned to the cation, $\mathrm{C}_{48} \mathrm{H}_{20}^{+}$. All the results are in agreement with previous observations (Chen et al. 2001). Band peak positions, intensities and FWHM are reported in Table 8.

\section{Comparison with the diffuse interstellar bands}

The position of the bands measured in the laboratory spectra of neutral and ionized PAHs are compared with DIB data in Table 9. The comparison is limited to band peak positions and relative band peak intensities since the interaction with the solid matrix leads to a broadening of intrinsic band profiles that 
Table 4. Absorption features associated with the UV/Vis/NIR spectra of neutral and ionized decacyclene $\left(\mathrm{C}_{36} \mathrm{H}_{18}\right)$ isolated at $4 \mathrm{~K}$ in inert-gas matrices.

\begin{tabular}{|c|c|c|c|c|c|c|c|c|}
\hline \multicolumn{3}{|c|}{ Neutral in neon matrix } & \multicolumn{3}{|c|}{10 min. irr. in neon matrix } & \multicolumn{2}{|c|}{10 min. irr. in neon/ $\mathrm{NO}_{2}$ matrix } & \multirow[b]{2}{*}{ Assignment } \\
\hline $\begin{array}{l}\text { Peak pos. } \\
(\mathrm{nm})\end{array}$ & $\begin{array}{c}F W H M \\
(\mathrm{~nm})\end{array}$ & $A b s_{\max }$ & $\begin{array}{c}\text { Peak pos. } \\
(\mathrm{nm})\end{array}$ & $\begin{array}{c}F W H M \\
(\mathrm{~nm})\end{array}$ & $A b s_{\max }$ & $\begin{array}{c}F W H M \\
(\mathrm{~nm})\end{array}$ & $A b s_{\max }$ & \\
\hline $213.51(\mathrm{~B})$ & 25.3 & 0.414 & 532.92 & 35.4 & 0.010 & 34.2 & 0.026 & + \\
\hline $251.78(\mathrm{~B})$ & 20.8 & 0.204 & 814.67 & 42.9 & 0.008 & 64.5 & 0.024 & + \\
\hline $275.30(\mathrm{~B})$ & 4.1 & 0.148 & 1083.32 & 74.8 & 0.010 & 53.9 & 0.022 & + \\
\hline 284.79 (B) & 5.5 & 0.148 & & & & & & \\
\hline $297.14(\mathrm{~B})$ & 7.8 & 0.110 & & & & & & \\
\hline $310.67(\mathrm{~B})$ & 5.1 & 0.153 & & & & & & \\
\hline $326.42(\mathrm{~B})$ & 3.6 & 0.219 & & & & & & \\
\hline $346.54(\mathrm{~B})$ & 7.9 & 0.224 & & & & & & \\
\hline $364.21(\mathrm{~B})$ & 9.2 & 0.275 & & & & & & \\
\hline $392.20(\mathrm{~B})$ & 12.7 & 0.148 & & & & & & \\
\hline $401.51(\mathrm{~B})$ & 1.9 & 0.114 & & & & & & \\
\hline $406.01(\mathrm{~B})$ & 4.1 & 0.116 & & & & & & \\
\hline $410.51(\mathrm{~B})$ & 1.5 & 0.111 & & & & & & \\
\hline $412.00(\mathrm{~B})$ & 0.3 & 0.110 & & & & & & \\
\hline 414.82 (B) & 0.8 & 0.122 & & & & & & \\
\hline $419.02(\mathrm{~B})$ & 4.2 & 0.085 & & & & & & \\
\hline 424.15 (B) & 4.6 & 0.055 & & & & & & \\
\hline 428.72 (B) & 0.8 & 0.047 & & & & & & \\
\hline 433.53 (B) & 1.0 & 0.014 & & & & & & \\
\hline 439.01 (B) & 1.0 & 0.007 & & & & & & \\
\hline 443.78 (B) & 0.8 & 0.051 & & & & & & \\
\hline 448.84 (B) & 1.0 & 0.018 & & & & & & \\
\hline $454.52(\mathrm{~B})$ & 0.9 & 0.010 & & & & & & \\
\hline
\end{tabular}

(B) = Blended peak. Assignment only apply to the peaks that are detected in the spectra of the irradiated sample. $+=$ cation.
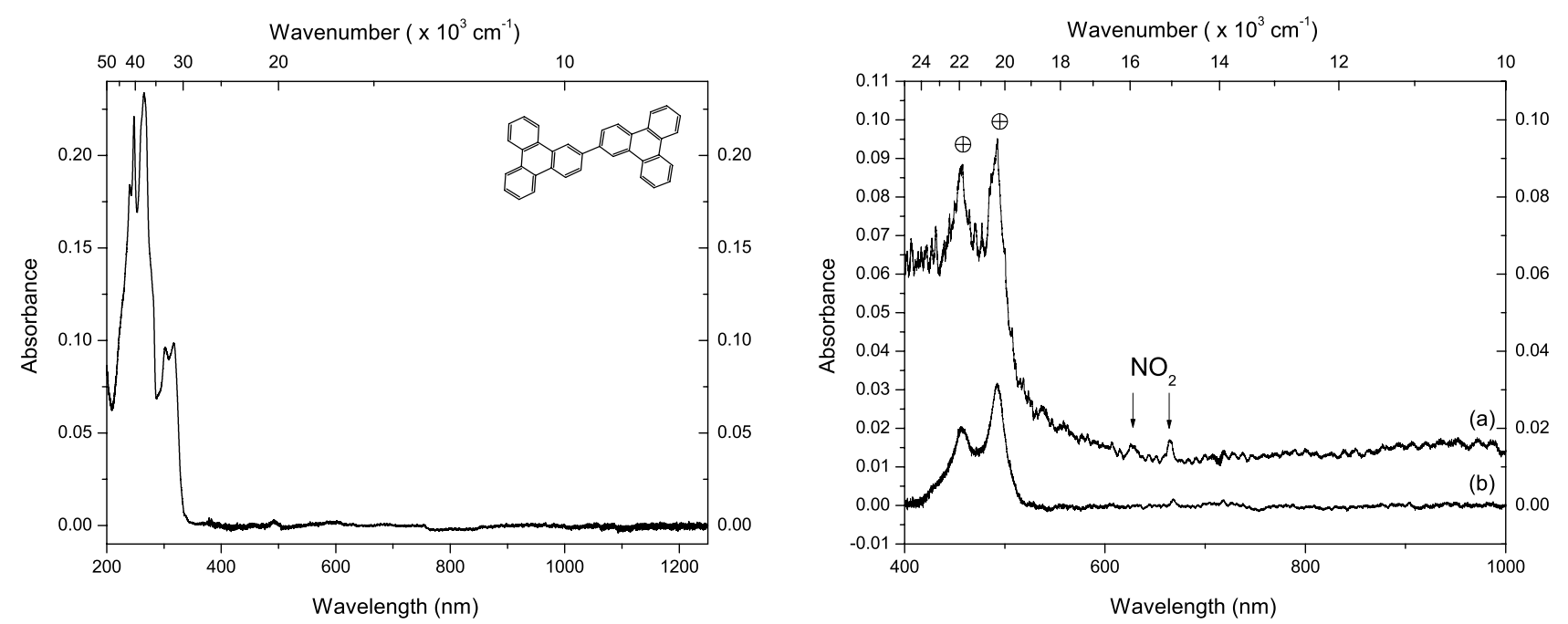

Fig. 6. Comparison of the spectral features associated with neutral (left panel) and VUV-irradiated (right panel) 2,2'-bis-triphenylyl (Tp) isolated in neon $(\mathrm{Ne})$ and in $\mathrm{Ne} / \mathrm{NO}_{2}$ matrices. a) is the spectrum of the photolysis products in a neon/ $/ \mathrm{NO}_{2}$ matrix; b) is the spectrum of the photolysis products in a neon matrix. Band peak positions, intensities and FWHM are given in Table 5.

prevents further comparison with astronomical data (Salama 1996; Salama et al. 1999). In Table 9 we have applied the criteria that have been used in our previous studies (Salama et al. 1999) when comparing MIS laboratory spectra with astronomical data. In the case of neutral PAHs we have taken into account all DIBs that fall in a window of $\sim 0.25 \%$ in fractional energy to the blue of the PAH band peak energy as measured in the matrix. In the case of ionized PAHs, we have taken into account all DIBs that fall in a window of $\sim 0.5 \%$ in fractional energy to the blue of the PAH ion band peak energy as measured in the matrix.

These criteria have been chosen to take into account the upper limit of the energy shift that is expected to be induced by the solid matrix environment on isolated PAH molecules and ions 
Table 5. Absorption features associated with the UV/Vis/NIR spectra of neutral and ionized 2, $2^{\prime}$-bis-triphenylyl $\left(\mathrm{C}_{36} \mathrm{H}_{22}\right)$, isolated at $4 \mathrm{~K}$ in inert-gas matrices.

Neutral in neon matrix 10 min. irr. in neon matrix 10 min. irr. in neon/ $/ \mathrm{NO}_{2}$ matrix

\begin{tabular}{lcccccccc}
\hline \hline $\begin{array}{l}\text { Peak pos. } \\
(\mathrm{nm})\end{array}$ & $\begin{array}{c}F W H M \\
(\mathrm{~nm})\end{array}$ & $A b s_{\max }$ & $\begin{array}{c}\text { Peak pos. } \\
(\mathrm{nm})\end{array}$ & $\begin{array}{c}F W H M \\
(\mathrm{~nm})\end{array}$ & $A b s_{\max }$ & $\begin{array}{c}F W H M \\
(\mathrm{~nm})\end{array}$ & $A b s_{\max }$ & Assignment \\
\hline $247.45(\mathrm{~B})$ & 3.5 & 0.219 & 457.69 & 33.3 & 0.018 & 31.6 & 0.034 & + \\
$263.97(\mathrm{~B})$ & 21.0 & 0.228 & 490.39 & 16.4 & 0.027 & 15.1 & 0.051 & + \\
$303.61(\mathrm{~B})$ & 17.5 & 0.095 & & & & & & \\
$315.55(\mathrm{~B})$ & 17.6 & 0.082 & & & & & & \\
\hline
\end{tabular}

(B) $=$ Blended peak. Assignments only apply to the peaks that are detected in the spectra of the irradiated sample. $+=$ cation.
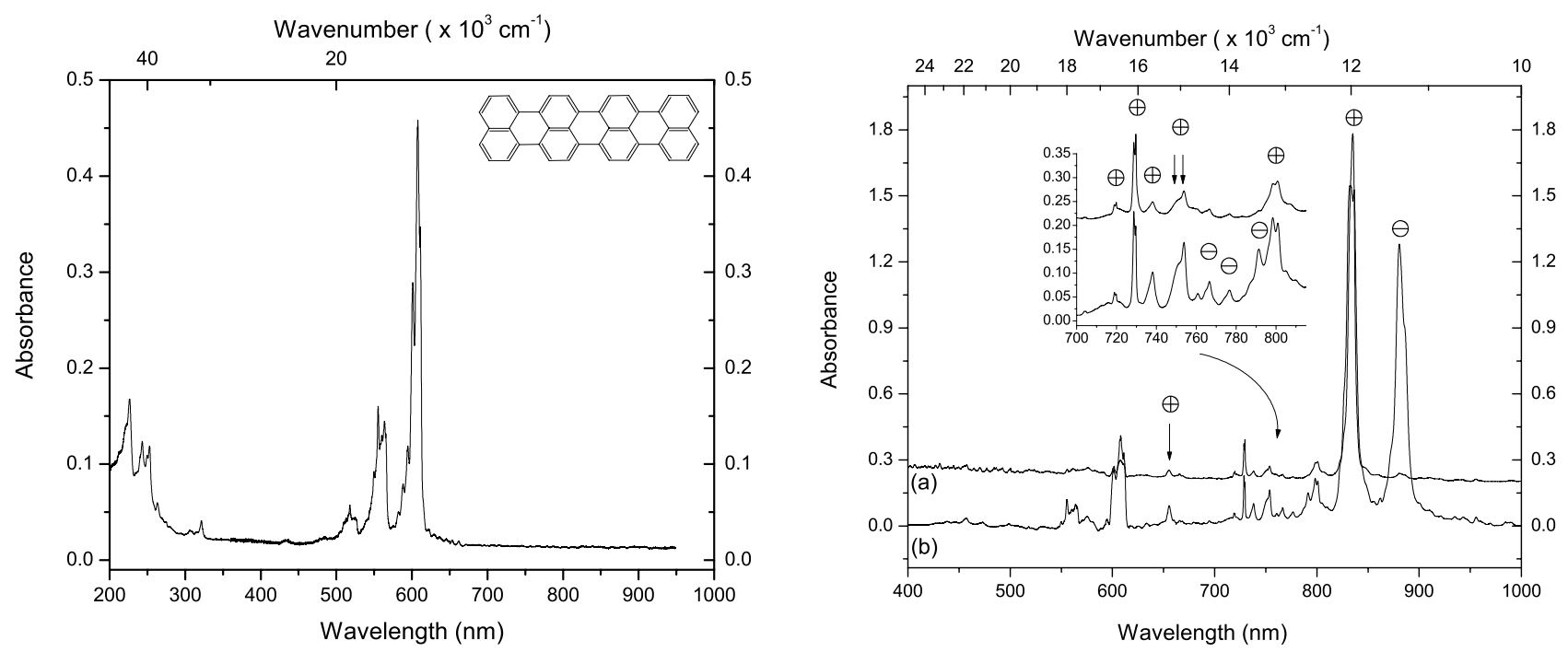

Fig. 7. Comparison of the spectral features associated with neutral (left panel) and VUV-irradiated (right panel) quaterrylene (Qt) isolated in neon $(\mathrm{Ne})$ and in $\mathrm{Ne} / \mathrm{NO}_{2}$ matrices. a) is the spectrum of the photolysis products in a neon $/ \mathrm{NO}_{2}$ matrix; b) is the spectrum of the photolysis products including, $\mathrm{Qt}^{+}$and $\mathrm{Qt}^{-}$, in a neon matrix. Band peak positions, intensities and $F W H M$ are given in Table 6.
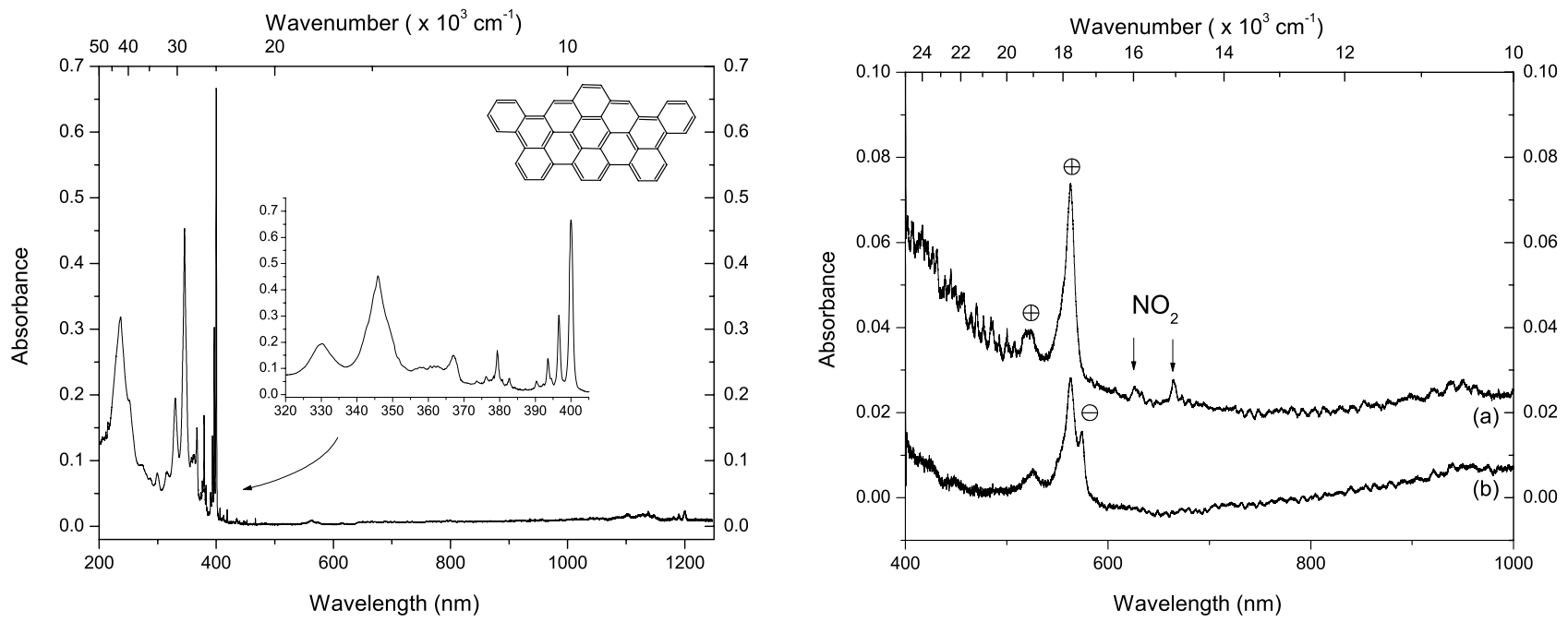

Fig. 8. Comparison of the spectral features associated with neutral (left panel) and VUV-irradiated (right panel) hexabenzo (a, cd, fgh, jk, m, qrs)peropyrene ( $\mathrm{Hp}$ ) isolated in neon $(\mathrm{Ne})$ and in $\mathrm{Ne} / \mathrm{NO}_{2}$ matrices. a) is the spectrum of the photolysis products in a neon $/ \mathrm{NO}_{2}$ matrix; b) is the spectrum of the photolysis products, including $\mathrm{Hp}^{+}$and $\mathrm{Hp}^{-}$, in a neon matrix. Band peak positions, intensities and $F W H M$ are reported in Table 7. 
Table 6. Absorption features associated with the UV/Vis/NIR spectra of neutral and ionized quaterrylene $\left(\mathrm{C}_{40} \mathrm{H}_{20}\right)$ isolated at $4 \mathrm{~K}$ in inert matrices.

Neutral in neon matrix 10 min. irr. in neon matrix 10 min. irr. in neon/ $\mathrm{NO}_{2}$ matrix

\begin{tabular}{|c|c|c|c|c|c|c|c|c|}
\hline $\begin{array}{l}\text { Peak pos. } \\
(\mathrm{nm})\end{array}$ & $\begin{array}{c}F W H M \\
(\mathrm{~nm})\end{array}$ & $A b s_{\max }$ & $\begin{array}{l}\text { Peak pos. } \\
\quad(\mathrm{nm})\end{array}$ & $\begin{array}{c}F W H M \\
(\mathrm{~nm})\end{array}$ & $A b s_{\max }$ & $\begin{array}{c}F W H M \\
(\mathrm{~nm})\end{array}$ & $A b s_{\max }$ & Assignment \\
\hline 206.41 & 81.9 & 0.080 & 456.44 & 4.5 & 0.034 & 10.9 & 0.275 & + \\
\hline 213.02 & 3.9 & 0.010 & 471.13 & 10.0 & 0.014 & & & - \\
\hline 221.75 & 7.0 & 0.043 & 655.56 & 2.9 & 0.091 & 3.3 & 0.053 & + \\
\hline 226.76 & 3.5 & 0.059 & 729.00 & 1.5 & 0.220 & 1.8 & 0.175 & + \\
\hline 244.57 & 11.4 & 0.025 & 737.68 & 3.2 & 0.096 & 3.1 & 0.047 & + \\
\hline 242.81 & 3.4 & 0.021 & 750.88 & 7.4 & 0.104 & 3.8 & 0.020 & + \\
\hline 249.57 & 2.4 & 0.022 & 753.90 & 0.3 & 0.098 & 3.3 & 0.040 & + \\
\hline 252.93 & 2.7 & 0.046 & 760.56 & 2.9 & 0.020 & 4.0 & 0.018 & + \\
\hline 263.50 & 2.7 & 0.014 & 764.65 & 2.0 & 0.017 & 2.8 & 0.008 & + \\
\hline 307.34 & 5.5 & 0.006 & 766.64 & 2.3 & 0.044 & & & - \\
\hline 315.59 & 5.2 & 0.005 & 776.39 & 2.7 & 0.063 & & & - \\
\hline 321.47 & 3.6 & 0.016 & 791.55 & 4.1 & 0.143 & & & - \\
\hline 433.60 & 13.2 & 0.006 & 798.97 & 5.7 & 0.209 & 5.7 & 0.091 & + \\
\hline 516.55 & 13.5 & 0.015 & 833.66 & 9.0 & 1.629 & 5.7 & 1.577 & + \\
\hline 517.96 & 1.7 & 0.006 & 881.84 & 9.5 & 1.223 & & & - \\
\hline 525.33 & 4.4 & 0.005 & 939.16 & 9.7 & 0.010 & 9.7 & 0.010 & + \\
\hline 540.59 & 8.6 & 0.003 & 955.94 & 4.2 & 0.012 & 4.2 & 0.012 & + \\
\hline 551.59 & 8.5 & 0.052 & 990.13 & 4.4 & 0.007 & 4.4 & 0.007 & + \\
\hline 555.26 & 1.8 & 0.048 & 1030.56 & 4.0 & 0.097 & 4.1 & 0.039 & + \\
\hline 560.85 & 7.7 & 0.084 & 1073.03 & 3.5 & 0.103 & 3.7 & 0.054 & + \\
\hline 564.98 & 3.2 & 0.050 & & & & & & \\
\hline 582.33 & 7.0 & 0.031 & & & & & & \\
\hline 588.02 & 3.4 & 0.045 & & & & & & \\
\hline 594.67 & 6.0 & 0.096 & & & & & & \\
\hline 600.74 & 3.3 & 0.226 & & & & & & \\
\hline 607.88 & 6.2 & 0.420 & & & & & & \\
\hline 611.65 & 1.3 & 0.100 & & & & & & \\
\hline 621.57 & 6.3 & 0.016 & & & & & & \\
\hline 629.54 & 4.6 & 0.009 & & & & & & \\
\hline 636.59 & 6.4 & 0.008 & & & & & & \\
\hline 645.88 & 6.2 & 0.006 & & & & & & \\
\hline 653.68 & 3.2 & 0.005 & & & & & & \\
\hline 661.77 & 4.1 & 0.005 & & & & & & \\
\hline
\end{tabular}

Assignments only apply to the peaks that are detected in the spectra of the irradiated sample. $+=$ cation; - = anion.
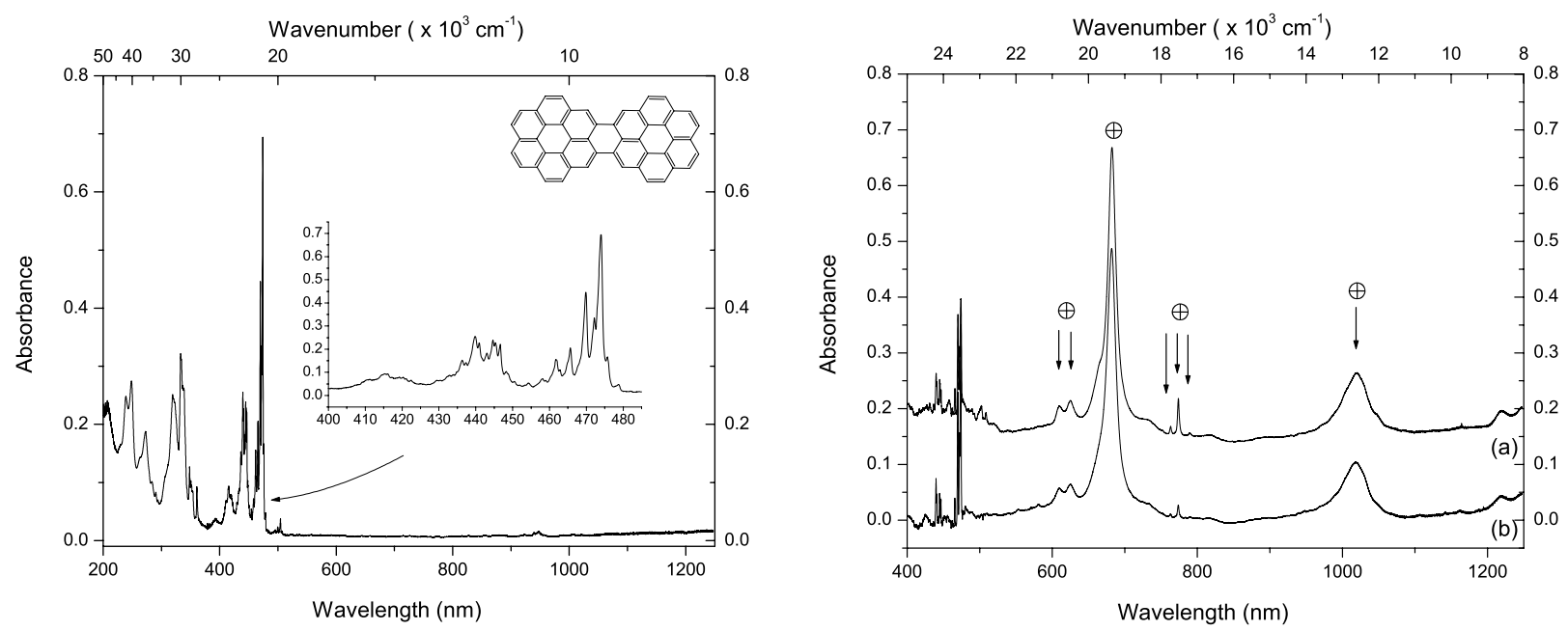

Fig. 9. Comparison of the spectral features associated with neutral (left panel) and VUV-irradiated (right panel) dicoronylene (Dc) isolated in neon $(\mathrm{Ne})$ and in $\mathrm{Ne} / \mathrm{NO}_{2}$ matrices. a) is the spectrum of the photolysis products in a neon $/ \mathrm{NO}_{2}$ matrix; $\mathbf{b}$ ) is the spectrum of the photolysis products in a neon matrix. Band peak positions, intensities and FWHM are given in Table 8. 
Table 7. Absorption features associated with the UV/Vis/NIR spectra of neutral and ionized hexabenzoperopyrene $\left(\mathrm{C}_{44} \mathrm{H}_{20}\right)$, at $4 \mathrm{~K}$ in inert-gas matrices.

\begin{tabular}{lcccccccc}
\multicolumn{2}{c}{ Neutral in neon matrix } & \multicolumn{1}{c}{10 min. irr. in neon matrix } & 10 min. irr. in neon/ $\mathrm{NO}_{2}$ matrix & \\
\hline \hline $\begin{array}{l}\text { Peak pos. } \\
(\mathrm{nm})\end{array}$ & $\begin{array}{c}F W H M \\
(\mathrm{~nm})\end{array}$ & $A b s_{\max }$ & $\begin{array}{c}\text { Peak pos. } \\
(\mathrm{nm})\end{array}$ & $\begin{array}{c}F W H M \\
(\mathrm{~nm})\end{array}$ & $A b s_{\max }$ & $\begin{array}{c}F W H M \\
(\mathrm{~nm})\end{array}$ & $A b s_{\max }$ & Assignment \\
\hline $236.02(\mathrm{~B})$ & 21.3 & 0.298 & 525.63 & 21.3 & 0.006 & 19.4 & 0.01 & + \\
$299.38(\mathrm{~B})$ & 4.1 & 0.081 & 562.47 & 17.2 & 0.028 & 10.3 & 0.045 & + \\
$315.99(\mathrm{~B})$ & 5.8 & 0.084 & 574.83 & 3.0 & 0.009 & & \\
$330.26(\mathrm{~B})$ & 6.0 & 0.189 & & & & & \\
$345.87(\mathrm{~B})$ & 5.3 & 0.411 & & & & & \\
$357.97(\mathrm{~B})$ & 2.9 & 0.104 & & & & & \\
$361.81(\mathrm{~B})$ & 6.2 & 0.108 & & & & & \\
$366.72(\mathrm{~B})$ & 3.9 & 0.142 & & & & & \\
373.67 & 0.8 & 0.051 & & & & & \\
376.28 & 0.7 & 0.068 & & & & & \\
379.35 & 0.7 & 0.158 & & & & \\
382.65 & 0.6 & 0.060 & & & & \\
390.38 & 0.5 & 0.052 & & & & \\
393.57 & 0.6 & 0.136 & & & & \\
396.63 & 0.6 & 0.294 & & & & \\
400.02 & 1.0 & 0.675 & & & & \\
406.37 & 0.7 & 0.028 & & & & \\
412.65 & 0.3 & 0.017 & & & & \\
418.71 & 0.7 & 0.021 & & & & \\
435.11 & 1.5 & 0.011 & & & & \\
1180.70 & 2.0 & 0.014 & & & & \\
1189.90 & 2.2 & 0.018 & & & & \\
1199.90 & 3.5 & 0.022 & & & & \\
\hline
\end{tabular}

B) = Blended peak. Assignment only apply to the peaks that are detected in the spectra of the irradiated sample. $+=$ cation; $-=$ anion.

(Salama 1996; Romanini et al. 1999). The estimate of the blue shift in peak position induced by the matrix - as opposed to the gas phase - is based on the limited information that is currently available on the spectra of PAH ions in the gas phase (Romanini et al. 1999; Bréchignac \& Pino 1999; Biennier et al. 2002). Of all the available DIB data we have deliberately chosen to only use data from one astronomical object, HD183143. The reason for this choice is that this object, being the source of extensive studies by many independent observers, is the best studied DIB object and can thus be used for reliable comparisons with the laboratory data. Finally, note that the coincidences that are found between PAH bands and specific DIBs do not imply that detection can be secured. They provide, however, a powerful and unique tool to select potential DIB carrier molecules that can be subsequently tested in an unambiguous way through gas phase studies in a free jet environment that provide information on intrinsic band profiles.

\subsection{Comparison of diphenanthrofuran bands with the observed DIBs}

Neutral diphenanthrofuran absorption bands do not correspond to any known DIB. A comparison of the DIB positions with the spectra of the diphenanthrofuran ions indicates that the position of the strongest cation band that peaks at $4444 \AA$ in matrix (predicted near $4422 \AA$ in the gas phase) comes close to the DIB $\lambda$ 4427.96. Six weaker DIBs: $\lambda$ 6302.29, $\lambda$ 6308.92, $\lambda 6311.53, \lambda 6317.75, \lambda 6324.81$ and $\lambda 6330.14$, come also close to the second strongest absorption of the diphenanthrofuran cation that peaks at $6332 \AA$ (predicted near $6300 \AA$ in the gas phase). Of these 6 DIBs only the first two are within $10 \AA$ of the calculated gas-phase peak positions.

The relative intensities of the two stronger cation absorption bands are $4444: 6332 \AA=1: 0.96$. None of the six DIBs that fall close to the $6332 \AA$ absorption band of the diphenanthrofuran cation presents the required intensity to match the band ratio measured in the laboratory. For example, the relative intensities of the two DIBs that most closely match the expected gas-phase positions are 4427.96: 6302.29 $\AA=1: 0.04$. Thus, despite the close agreement found in wavelength positions between the two stronger bands of the diphenanthrofuran cation and two DIBs, it is clear that the relative intensities differ considerably, ruling out this PAH ion as potential DIB carrier. (Note that the relative band intensities associated with specific PAH species are similar in solid matrices and in the gas phase (Romanini et al. 1999; Bréchignac \& Pino 1999)).

Finally, we note that the weak absorption that is associated with the diphenanthrofuran anion and that is measured at $7528 \AA$ (predicted at $7491 \AA$ in the gas phase) comes close to the weak $7493 \AA$ DIB.

\subsection{Comparison of terrylene bands with the observed DIBs}

Neutral terrylene absorption bands do not correspond to any known DIB. The two stronger features of the terrylene cation 
Table 8. Absorption features associated with the UV/Vis/NIR spectra of neutral and ionized dicoronylene $\left(\mathrm{C}_{48} \mathrm{H}_{20}\right)$, isolated at $4 \mathrm{~K}$ in inert-gas matrices.

\begin{tabular}{|c|c|c|c|c|c|c|c|c|}
\hline \multicolumn{3}{|c|}{ Neutral in neon matrix } & \multicolumn{3}{|c|}{10 min. irr. in neon matrix } & \multicolumn{2}{|c|}{$10 \mathrm{~min}$. irr. in neon/ $/ \mathrm{NO}_{2}$ matrix } & \multirow[b]{2}{*}{ Assignment } \\
\hline $\begin{array}{l}\text { Peak pos. } \\
(\mathrm{nm})\end{array}$ & $\begin{array}{c}F W H M \\
(\mathrm{~nm})\end{array}$ & $A b s_{\max }$ & $\begin{array}{l}\text { Peak pos. } \\
\text { (nm) }\end{array}$ & $\begin{array}{c}F W H M \\
(\mathrm{~nm})\end{array}$ & $A b s_{\max }$ & $\begin{array}{c}F W H M \\
(\mathrm{~nm})\end{array}$ & $A b s_{\max }$ & \\
\hline $238.80(\mathrm{~B})$ & 5.9 & 0.249 & 610.01 & 9.3 & 0.055 & 7.8 & 0.050 & + \\
\hline $248.30(\mathrm{~B})$ & 6.4 & 0.276 & 625.18 & 12.6 & 0.063 & 14.9 & 0.060 & + \\
\hline $272.81(\mathrm{~B})$ & 8.2 & 0.187 & 664.30 & 15.0 & 0.126 & 13.3 & 0.124 & + \\
\hline 283.26 (B) & 6.3 & 0.102 & 682.46 & 12.8 & 0.456 & 12.1 & 0.485 & + \\
\hline $290.21(\mathrm{~B})$ & 2.4 & 0.082 & 762.63 & 3.9 & 0.010 & 2.5 & 0.014 & + \\
\hline 293.77 (B) & 1.8 & 0.069 & 773.71 & 2.7 & 0.025 & 2.6 & 0.064 & + \\
\hline 306.67 (B) & 6.9 & 0.103 & 1017.1 & 26.3 & 0.103 & 34.0 & 0.109 & + \\
\hline $319.20(\mathrm{~B})$ & 11.9 & 0.251 & & & & & & \\
\hline 333.33 (B) & 4.3 & 0.317 & & & & & & \\
\hline 338.64 (B) & 4.4 & 0.247 & & & & & & \\
\hline 348.14 (B) & 1.2 & 0.127 & & & & & & \\
\hline 350.99 (B) & 8.0 & 0.103 & & & & & & \\
\hline 360.88 (B) & 1.2 & 0.092 & & & & & & \\
\hline 392.80 & 8.6 & 0.036 & & & & & & \\
\hline 436.23 & 0.8 & 0.150 & & & & & & \\
\hline 437.43 & 0.9 & 0.139 & & & & & & \\
\hline 439.74 & 1.2 & 0.254 & & & & & & \\
\hline 440.98 & 0.6 & 0.226 & & & & & & \\
\hline 442.95 & 0.6 & 0.182 & & & & & & \\
\hline 444.60 & 0.7 & 0.240 & & & & & & \\
\hline 445.39 & 0.5 & 0.225 & & & & & & \\
\hline 446.55 & 0.8 & 0.220 & & & & & & \\
\hline 450.39 & 1.0 & 0.060 & & & & & & \\
\hline 454.30 & 1.0 & 0.053 & & & & & & \\
\hline 458.03 & 0.7 & 0.072 & & & & & & \\
\hline 458.99 & 0.4 & 0.064 & & & & & & \\
\hline 461.78 & 0.7 & 0.158 & & & & & & \\
\hline 462.95 & 0.7 & 0.112 & & & & & & \\
\hline 465.74 & 0.6 & 0.204 & & & & & & \\
\hline 469.74 & 0.8 & 0.438 & & & & & & \\
\hline 472.15 & 1.0 & 0.333 & & & & & & \\
\hline 473.85 & 0.9 & 0.698 & & & & & & \\
\hline 495.02 & 1.6 & 0.018 & & & & & & \\
\hline 499.62 & 1.1 & 0.023 & & & & & & \\
\hline 504.18 & 1.3 & 0.035 & & & & & & \\
\hline
\end{tabular}

(B) $=$ Blended peak. Assignments only apply to the peaks that are detected in the spectra of the irradiated sample. $+=$ cation.

fall at 6904 and $7039 \AA$ with a relative intensity of $1: 0.2$. The predicted positions for these 2 bands in the gas phase are 6870 and $7004 \AA$ respectively. The closest counterparts in the DIB spectrum fall at 6886.92 and $7004.30 \AA$, respectively, with a relative intensity of $1: 0.07$. This close correlation -within the uncertainties attached to the astronomical measurements of weak DIBs- makes the terrylene cation a promising candidate for further gas-phase studies.

Finally, the strongest $(0.722 \mathrm{Abs})$ anion feature measured at $7413 \AA$ in the laboratory (predicted near $7376 \AA$ in the gas phase) coincides with the $\lambda 7375.90$ DIB. The two other, weaker, spectral features associated with the anion and measured in the laboratory at 9015 and $10012 \AA$, respectively, are of similar intensity ( 0.086 and $0.088 \mathrm{Abs}$, respectively) with a relative intensity of 1 : 0.12 with the strongest, $7413 \AA$, anion band. Any DIBs correlated with these 2 bands would thus be expected to be found at the $0.002 \mathrm{Abs}$ level that is barely at the detection limit. Moreover, the 2 bands fall in a wavelength region that is astronomically not well explored. We thus conclude that the case is still open and that, if further verified, only the $\lambda 7375.90$ DIB might be correlated with the terrylene anion, the wavelength agreement that is observed between the $10012 \AA$ laboratory band with the $\lambda 9955.90$ DIB being purely coincidental.

\subsection{Comparison of ovalene bands with the observed DIBs}

In this case, the measurements were compared with the spectrum previously published by Ehrenfreund et al. (1992). Some discrepancy was found in the case of the neutral molecule. Comparison of the spectral data between the two studies 
Table 9. Comparison of confirmed DIBs towards HD183143 with band peak positions of neutral (0) PAHs and PAH cations (+) and anions (-).

\begin{tabular}{|c|c|c|c|c|c|}
\hline $\begin{array}{l}\lambda \text { PAH } \\
(\AA)\end{array}$ & $A b s_{\max }$ & $\begin{array}{c}\lambda \mathrm{PAH} \text { (gas) } \\
(\AA)\end{array}$ & $\begin{array}{c}\lambda \mathrm{HD} 183143 \\
(\AA)\end{array}$ & $A b s_{\max }$ & Ref. \\
\hline 4444 DiFurA+ & 0.055 & 4422 & 4427.96 & 0.141 & BB37 \\
\hline \multirow[t]{6}{*}{6332 DiFurB+ } & 0.053 & 6300 & 6302.29 & 0.006 & ОТ00 \\
\hline & & & 6308.92 & 0.038 & HL91 \\
\hline & & & 6311.53 & 0.017 & $\mathrm{He} 75$ \\
\hline & & & 6317.75 & 0.020 & JD93 \\
\hline & & & 6324.81 & 0.018 & JD93 \\
\hline & & & 6330.14 & 0.018 & JD93 \\
\hline 7528 DiFurA- & 0.006 & 7491 & 7492.93 & 0.004 & VF02 \\
\hline 4848 TerC0 & 0.328 & 4836 & & & \\
\hline 5135 TerD0 & 0.314 & 5122 & & & \\
\hline 5198 TerB0 & 0.637 & 5185 & & & \\
\hline 5263 TerA0 & 0.951 & 5250 & & & \\
\hline \multirow[t]{5}{*}{6646 TerE+ } & 0.123 & 6613 & 6613.63 & 0.075 & Me34 \\
\hline & & & 6622.59 & 0.006 & K95 \\
\hline & & & 6632.86 & 0.007 & JD94 \\
\hline & & & 6635.50 & 0.003 & K95 \\
\hline & & & 6644.33 & 0.009 & ОТ00 \\
\hline \multirow[t]{7}{*}{6707 TerC+ } & 0.293 & 6674 & 6684.83 & 0.002 & OT00 \\
\hline & & & 6686.46 & 0.002 & ОТ00 \\
\hline & & & 6689.38 & 0.008 & K95 \\
\hline & & & 6694.40 & 0.004 & JD94 \\
\hline & & & 6699.28 & 0.023 & Sa78 \\
\hline & & & 6701.87 & 0.009 & Fe83 \\
\hline & & & 6707.73 & 0.002 & ОТ00 \\
\hline 6904 TerA+ & 1.908 & 6870 & 6886.92 & 0.073 & HL91 \\
\hline 7039 TerB+ & 0.358 & 7004 & 7004.30 & 0.005 & VF02 \\
\hline \multirow[t]{6}{*}{7413 TerA- } & 0.722 & 7376 & 7375.90 & 0.017 & JD94 \\
\hline & & & 7385.92 & 0.026 & JD94 \\
\hline & & & 7391.48 & 0.003 & VF02 \\
\hline & & & 7401.71 & 0.015 & JD94 \\
\hline & & & 7405.77 & 0.014 & JD94 \\
\hline & & & 7406.35 & 0.017 & JD94 \\
\hline 8704 TerD+ & 0.162 & 8661 & & & \\
\hline 9015 TerC- & 0.086 & 8970 & & & \\
\hline $9667 \mathrm{TerF}+$ & 0.091 & 9619 & & & \\
\hline \multirow[t]{2}{*}{10012 TerB- } & 0.088 & 9962 & 9955.90 & 0.043 & VF02 \\
\hline & & & 9971.07 & 0.028 & VF02 \\
\hline $4256 \mathrm{OvaC0}$ & 0.105 & 4246 & & & \\
\hline 4305 OvaA0 & 0.701 & 4294 & & & \\
\hline $4543 \mathrm{OvaF}+$ & 0.010 & 4520 & & & \\
\hline 4632 OvaA+ & 0.097 & 4609 & & & \\
\hline 719 OvaE+ & 0.010 & 4696 & & & \\
\hline \multirow[t]{2}{*}{5260 OvaD+ } & 0.011 & 5234 & 5236.34 & 0.030 & ОТ00 \\
\hline & & & 5247.91 & 0.015 & OT00 \\
\hline 5357 OvaA- & 0.015 & 5330 & 5349.16 & 0.007 & OT00 \\
\hline \multirow[t]{4}{*}{$5621 \mathrm{OvaC}+$} & 0.014 & 5593 & 5594.54 & 0.012 & ОТ00 \\
\hline & & & 5597.38 & 0.002 & ОТ00 \\
\hline & & & 5600.49 & 0.008 & OT00 \\
\hline & & & 5609.96 & 0.011 & JD94 \\
\hline 8082 OvaI+ & 0.003 & 8042 & & & \\
\hline 8574 OvaH+ & 0.006 & 8531 & 8530.79 & 0.04 & JD94 \\
\hline 8696 OvaG+ & 0.008 & 8652 & & & \\
\hline 9749 OvaB+ & 0.031 & 9701 & & & \\
\hline
\end{tabular}


Table 9. continued.

\begin{tabular}{|c|c|c|c|c|c|}
\hline $\begin{array}{l}\lambda \text { PAH } \\
(\AA)\end{array}$ & $A b s_{\max }$ & $\begin{array}{c}\lambda \mathrm{PAH} \text { (gas) } \\
(\AA)\end{array}$ & $\begin{array}{c}\lambda \mathrm{HD} 183143 \\
(\AA)\end{array}$ & $A b s_{\max }$ & Ref. \\
\hline 5329 DecaA+ & 0.010 & 5303 & & & \\
\hline 8147 DecaB+ & 0.008 & 8106 & 8104.89 & 0.017 & VF02 \\
\hline $10833 \mathrm{DecaC}+$ & 0.010 & 10779 & & & \\
\hline 4577 TPhenB+ & 0.012 & 4554 & & & \\
\hline 4904 TPhenA+ & 0.019 & 4880 & 4882.56 & 0.064 & Wi58 \\
\hline \multirow[t]{4}{*}{5609 QuaTC0 } & 0.084 & 5595 & 5594.54 & 0.012 & OT00 \\
\hline & & & 5597.38 & 0.002 & OT00 \\
\hline & & & 5600.49 & 0.020 & OT00 \\
\hline & & & 5609.96 & 0.010 & JD94 \\
\hline \multirow[t]{2}{*}{5947 QuaTB0 } & 0.096 & 5932 & 5945.53 & 0.012 & K95 \\
\hline & & & 5947.29 & 0.017 & K95 \\
\hline \multirow[t]{3}{*}{6079 QuaTA0 } & 0.420 & 6063 & 6065.19 & 0.017 & JD94 \\
\hline & & & 6068.45 & 0.005 & ОТ00 \\
\hline & & & 6071.08 & 0.007 & JD94 \\
\hline 7290 QuaTB+ & 0.220 & 7254 & 7274.50 & 0.017 & JD94 \\
\hline 7509 QuaTD+ & 0.072 & 7471 & & & \\
\hline \multirow[t]{2}{*}{7916 QuaTB- } & 0.143 & 7876 & 7904.92 & 0.015 & VF02 \\
\hline & & & 7915.13 & 0.022 & JD94 \\
\hline \multirow[t]{2}{*}{7990 QuaTC+ } & 0.209 & 7950 & 7951.25 & 0.010 & VF02 \\
\hline & & & 7987.89 & 0.026 & JD94 \\
\hline 8337 QuaTA+ & 1.629 & 8295 & & & \\
\hline 8818 QuaTA- & 1.223 & 8775 & & & \\
\hline \multirow[t]{3}{*}{5256 HBenzE+ } & 0.006 & 5230 & 5236.34 & 0.026 & OT00 \\
\hline & & & 5247.91 & 0.015 & OT00 \\
\hline & & & 5349.16 & 0.007 & ОТ00 \\
\hline \multirow[t]{3}{*}{5625 HBenzA+ } & 0.028 & 5597 & 5597.38 & 0.002 & ОТ00 \\
\hline & & & 5600.49 & 0.008 & ОТ00 \\
\hline & & & 5609.96 & 0.011 & JD94 \\
\hline \multirow[t]{2}{*}{5748 HBenzB- } & 0.015 & 5720 & 5719.68 & 0.020 & JD94 \\
\hline & & & 5746.21 & 0.017 & JD94 \\
\hline 4398 DicoC0 & 0.254 & 4386 & & & \\
\hline 4446 DicoD0 & 0.240 & 4435 & & & \\
\hline 4630 DicoE0 & 0.112 & 4618 & & & \\
\hline 4657 DicoB0 & 0.438 & 4646 & & & \\
\hline \multirow[t]{2}{*}{4739 DicoA0 } & 0.698 & 4727 & 4726.35 & 0.063 & $\mathrm{He} 75$ \\
\hline & & & 4728.35 & 0.027 & $\mathrm{He} 75$ \\
\hline \multirow[t]{3}{*}{6100 DicoD+ } & 0.055 & 6070 & 6071.08 & 0.007 & JD94 \\
\hline & & & 6084.94 & 0.009 & ОТ00 \\
\hline & & & 6089.79 & 0.035 & HL91 \\
\hline \multirow[t]{8}{*}{6252 DicoC+ } & 0.063 & 6221 & 6220.86 & 0.008 & K95 \\
\hline & & & 6223.53 & 0.017 & JD94 \\
\hline & & & 6226.02 & 0.007 & K95 \\
\hline & & & 6234.01 & 0.023 & $\mathrm{He} 75$ \\
\hline & & & 6236.71 & 0.008 & JD93 \\
\hline & & & 6244.42 & 0.024 & OT00 \\
\hline & & & 6245.36 & 0.010 & K95 \\
\hline & & & 6250.77 & 0.006 & ОТ00 \\
\hline \multirow[t]{6}{*}{6825 DicoA+ } & 0.456 & 6791 & 6792.54 & 0.029 & $\mathrm{He} 88$ \\
\hline & & & 6795.18 & 0.016 & $\mathrm{He} 88$ \\
\hline & & & 6801.39 & 0.016 & $\mathrm{He} 88$ \\
\hline & & & 6804.82 & 0.004 & $\mathrm{He} 88$ \\
\hline & & & 6811.30 & 0.019 & $\mathrm{He} 88$ \\
\hline & & & 6812.39 & 0.019 & $\mathrm{He} 88$ \\
\hline \multirow[t]{5}{*}{7737 DicoE+ } & 0.025 & 7699 & 7702.32 & 0.003 & VF02 \\
\hline & & & 7705.90 & 0.024 & JD94 \\
\hline & & & 7709.67 & 0.017 & JD94 \\
\hline & & & 7713.49 & 0.014 & JD94 \\
\hline & & & 7721.03 & 0.048 & HL91 \\
\hline
\end{tabular}


Table 9. continued.

\begin{tabular}{lccccc}
\hline \hline $\begin{array}{l}\lambda \text { PAH } \\
(\AA)\end{array}$ & $A b s_{\max }$ & $\begin{array}{c}\lambda \text { PAH (gas) } \\
(\AA)\end{array}$ & $\begin{array}{c}\lambda \text { HD183143 } \\
(\AA)\end{array}$ & $A b s_{\max }$ & Ref. \\
\hline & & & 7727.95 & 0.005 & VF02 \\
& & & 7730.19 & 0.004 & VF02 \\
10171 DicoB+ & \multirow{2}{*}{0.103} & \multirow{2}{*}{10120} & 10130.33 & 0.066 & VF02 \\
& & & 10139.24 & 0.063 & VF02 \\
& & 10145.67 & 0.030 & VF02 \\
& & 10148.70 & 0.029 & VF02 \\
& & 10152.62 & 0.027 & VF02 \\
& & 10158.61 & 0.047 & VF02 \\
& & & 10164.55 & 0.042 & VF02 \\
\hline
\end{tabular}

$\lambda$ PAH (gas) corresponds to the laboratory wavelength that has been blue shifted by $0.25 \%$ and $0.5 \%$ in energy to account for the blue shift in energy that is expected for cold gas phase neutral and ionic PAHs, respectively, and is derived from the case studies of naphthalene (Salama \& Allamandola 1991; Romanini et al. 1999) and phenanthrene (Bréchignac \& Pino 1999). DiFur: diphenanthrofuran, $\mathrm{C}_{28} \mathrm{H}_{16} \mathrm{O}$; Ter: terrylene, $\mathrm{C}_{30} \mathrm{H}_{16}$; Ova: ovalene, $\mathrm{C}_{32} \mathrm{H}_{14}$; Deca: decacyclene, $\mathrm{C}_{36} \mathrm{H}_{18}$; TPhen: 2, 2'-bis- triphenylyl, $\mathrm{C}_{36} \mathrm{H}_{22}$; Quat: quaterrylene, $\mathrm{C}_{40} \mathrm{H}_{20}$; HBenz: hexabenzoperopyrene, $\mathrm{C}_{44} \mathrm{H}_{20}$; Dico: dicoronylene, $\mathrm{C}_{48} \mathrm{H}_{20}$. A = Strongest absorption band in the VUV-NIR range, $\mathrm{B}=$ second strongest absorption band, and so forth; $+=$ cation, - = anion, $0=$ neutral band. All listed DIB features are observed towards source HD183143 and taken from O'Tuairisg et al. 2000 for DIBs with $4000 \mathrm{~nm}<\lambda \leq 6812 \mathrm{~nm}$, Jenniskens et al. (1994) for DIBs with $4000 \mathrm{~nm}<\lambda<8648 \mathrm{~nm}$, and from Vuong $\&$ Foing (2002) for DIBs with $\lambda>7000 \mathrm{~nm}$. References indicate the original description of the features. $A b s_{\max }$ for DIBs is defined as the line center depth and is related to the equivalent width as $E W \sim F W H M \times$ center depth. The limit of detection of the center depth is $2.5 \times 10^{-3}$ for a $2 \AA$ wide DIB with $E W$ detection limit $5 \mathrm{~mA}$. Detailed discussion on errors in $E W$ values for DIBs can be found in O'Tuairisg et al. (2000) and in Cami et al. (1997). Reference codes: BB37- Beals \& Blanchet (1937), Fe83- Ferlet et al. (1983), GA00- Galazutdinov (2000), He67- Herbig (1967), He75- Herbig (1975), He88- Herbig (1988), HL91- Herbig \& Leka (1991), JD93- Jenniskens \& Deśert (1993), JD94- Jenniskens \& Deśert (1994), K95- Krelowski et al. (1995), Me34- Merril (1934), Sa78- Sanner et al. (1987), OT00- O’Tuairisg et al. (2000), VF02- Vuong \& Foing (2002), Wi58- Wilson (1958).

indicates that the data for neutral ovalene that are presented in this work exhibit additional, narrower, peaks. Since these peaks were unresolved in the Ehrenfreund et al. study we conclude that the spectrum that is presented here for the neutral molecule is more representative, and we thus base our conclusions on these most recent results. Total agreement is found between the two studies when comparing the spectra of the ion.

None of the neutral bands match any known DIBs. Similarly, the two strongest ovalene cation features have no DIB counterparts. Although the third and fourth strongest cation peaks come close to known DIBs, the associated relative intensities do not match the laboratory measurements lead to $5621: 5260 \AA=1: 0.8$ compared to $5594.54: 5236.34 \AA=$ $1: 2.5$ for the corresponding DIBs. These findings thus rule out the ovalene cation as a potential DIB carrier.

We note that the only absorption band that is associated to the anion in the laboratory spectra and measured at $5357 \AA$ (predicted at $5330 \AA$ in the gas phase) falls close to the weak $(0.007 A b s) \lambda 5349$ DIB. The strong cation band at $9749 \AA$ that is observed in the lab spectra is shifted $30 \AA$ compared to the measurement by Ehrenfreund et al. (1992) (which is probably due to saturation making an exact determination of the wavelength position impossible). However, there is no counterpart for this band in the DIB spectra (Ehrenfreund et al. 1995).

\subsection{Comparison of decacyclene bands with the observed DIBs}

None of the bands of neutral decacyclene match any known DIBs. The cation spectrum shows three broad absorption features of equal intensities (about $0.01 \mathrm{Abs}$ ). The band measured at $8147 \AA$ (predicted at $8106 \AA$ in the gas phase) coincides with the $\lambda 8104.89$ DIB. The poor quality of the laboratory spectrum in this case prohibits further conclusions and the case is pending future gas-phase experiments.

\subsection{Comparison of 2, 2' -bis-triphenylyl bands with the observed DIBs}

Neutral triphenylyl absorb in the UV where no DIBs have been detected. The two visible absorption features associated with the triphenylyl cation have relative intensities $4904: 4577 \AA=$ $1: 0.6$ and fall in the DIB region. The strongest of the two bands falls at $4904 \AA$ (4880 ̊ predicted gas-phase value) and is close to the $\lambda 4882.56$ DIB. No DIB counterpart is found, however, for the second strongest band that falls at $4577 \AA$ (4554 $\AA$ in the gas-phase). If the $\lambda 4882.56 \mathrm{DIB}(0.064 \mathrm{Abs}$ in HD183143) were indeed associated with the triphenyl cation, a second, weaker, DIB (0.038 Abs in HD183143) should be detected near $4550 \AA$. The absence of any DIB at this wavelength seems to rule out the triphenylyl cation as a potential DIB carrier.

\subsection{Comparison of quaterrylene bands with the observed DIBs}

Neutral quaterrylene absorbs in the DIB range. The strongest features of neutral quaterrylene measured in the MIS spectra fall at 6079,5947 and $5609 \AA$ with relative intensities = 1 : $0.23: 0.20$. The DIBs that most closely match the intensity ratio of these neutral features at the predicted gas phase wavelengths 
are $\lambda 6065.19, \lambda 5945.53$ and $\lambda 5597.38$ with relative intensities $=1: 0.7: 0.12$. This is marginally in agreement considering the uncertainties associated with DIB strength measurements.

No DIBs are found near the positions of the strongest cation and anion absorption features that fall at $8337 \AA$ and $8818 \AA$ respectively in Ne matrices $(8295 \AA$ and $8775 \AA$ respectively in the gas phase) making the quaterrylene cation and anion unlikely DIB carriers.

\subsection{Comparison of hexabenzoperopyrene bands with the observed DIBs}

None of the features associated with the neutral spectrum of hexabenzoperopyrene falls in the DIB region ( 4000-10 $000 \AA$ ). The three absorption bands associated with the ions ( 2 for the cation and 1 for the anion) fall, however, in the DIB range and can potentially be matched with eight DIBs when taking into account the projected peak positions in the gas phase. The two cation peaks are measured at $5625 \AA$ and $5256 \AA$ in Ne matrices with a relative intensity ratio $=1: 0.2$. Three DIBs are found in the wavelength window associated with the strongest cation band at $5625 \AA(5597 \AA$ projected value in the gas-phase). They are the $\lambda$ 5597.38, $\lambda 5600.49$ and $\lambda 5609.96$ DIBs with intensities of $0.002,0.008$ and $0.011 \mathrm{Abs}$, respectively. Thus, in the best scenario case, where the strongest of the 3 DIBs (the $\lambda 5609.96$ DIBs of $0.011 \mathrm{Abs}$ ) is correlated to the hexabenzoperopyrene cation, a DIB at the $0.002 \mathrm{Abs}$ level is expected near the position of the second strongest band of the cation, a level that is barely at the detection limit.

We thus conclude that the case is still open. Only one of the $\lambda$ 5597.38, $\lambda 5600.49$ and $\lambda 5609.96$ DIBs might be correlated with the hexabenzoperopyrene cation. The wavelength agreement that is observed between the $5256 \AA$ cation band and the $\lambda 5236.3, \lambda 5247.9$ and $\lambda 5349.1$ DIBs is purely coincidental. Thus, any correlation between the hexabenzoperopyrene cation and the DIBs must, if confirmed, be limited to the strongest cation band (5625 $\AA$ in MIS and $5597 \AA$ expected in the gas phase).

Finally, the only band associated with the hexabenzoperopyrene anion in the laboratory spectra falls at $5748 \AA$ (5720 in the gas phase) and can potentially be matched by either the $\lambda 5719.68$ or the $\lambda 5746.21 \mathrm{DIB}$.

\subsection{Comparison of dicoronylene bands with the observed DIBs}

The strongest absorption band of neutral dicoronylene measured at $4739 \AA$ in matrix (4727 $\AA$ projected value in the gas phase) comes close to two DIBs falling at 4726.35 and $4728.35 \AA$ respectively. No DIBs are found near the other strong absorption band of neutral dicoronylene making the neutral molecule an unlikely DIB carrier.

The strongest cation absorption band measured at $6825 \AA$ in matrix (6791 $\AA$ in the gas phase) dominates clearly the spectrum with an intensity of $0.456 \mathrm{Abs}$. Among all the 6 DIBs that potentially match this band in terms of peak position, the $\lambda 6792.54 \mathrm{DIB}(0.029 \mathrm{Abs})$ provides the closest correspondence. The band intensity ratio with the next strongest band of the dicoronylene cation (10171 $\AA$ in MIS) is 1:0.23. Thus any DIB correlated with the second strongest cation band is predicted to have intensity ranging from 0.007 (detectable if not obscured by other band) to 0.001 (below detection limit) Abs. It is clear that none of the 7 DIBs associated with the $10171 \AA$ band of the dicoronylene cation in Table 9 meets this requirement.

The wavelength agreement that is observed between $10171 \AA$ cation band and the DIBs detected around $1 \mu \mathrm{m}$ is purely coincidental. We also note that the spectral region around $1 \mu \mathrm{m}$ is not well explored in astronomical observations.

Thus, any assignment between the dicoronylene cation and the DIBs must, if confirmed, be limited to the strongest cation band (6825 $\AA$ in MIS and $6791 \AA$ in the gas phase).

\section{Conclusion}

Astronomical data obtained with the ISO satellite have provided evidence for the presence of PAHs of molecular sizes larger than was previously assumed ( $\sim 50 \mathrm{C}$ atoms) as well as 5-ring PAH structures (Tielens et al. 1999). We have therefore measured large $\left(\mathrm{C}_{28}-\mathrm{C}_{48}\right)$ PAHs, with condensed and open structures, including pentagons and heteroatoms, and measured the absorption spectra, in the UV-NIR range, of the neutral and ionized forms when isolated in cold $(5 \mathrm{~K})$, inert-gas, matrices.

The promising candidates for future gas-phase experiments are those neutral and ionized PAHs that possess one or more bands that match DIB features in the wavelength comparison window. In summary, the MIS laboratory data obtained in this work indicate that some large neutral and ionized PAH are promising candidates for the DIB carriers. Out of a laboratory set of 21 PAH species (including 8 neutral PAHs, 8 PAH cations and 5 PAH anions) which sample compact and non-compact PAHs, and PAHs with a heteroatom, 8 exhibit a possible coincidence with DIBs, 1 cannot be settled, and 12 can be excluded as potential DIB carriers. The gas-phase, jet, experiments which are now being developed (Biennier et al. 2002) will provide the much needed data to definitively assess the validity of the PAH proposal with regards to the DIBs. More astronomical surveys of DIB objects are also needed, especially in the wavelength ranges that have not been as yet frequently observed.

A few general remarks can be derived from this laboratory study of large PAHs. First, terrylene, ovalene, hexabenzoperopyrene, quaterrylene and dicoronylene each exhibits a strong cation absorption feature that clearly dominates the spectrum in the 400-1000 $\mathrm{nm}$ range. The observation of this spectral characteristic of PAH ions confirms the predictions that had been previously made for the PAH-DIB model based on the study of smaller-size PAHs (Salama et al. 1996). Similarly, a displacement toward the red is observed for the absorption of PAHs included within the same chemical subclass (e.g., perylene (Joblin et al. 1999), terrylene and quaterrylene). All these observations are useful to derive general properties of PAHs in the astrophysical context. For example, one can predict that larger PAHs in the linear series perylene, terrylene and quaterrylene will be difficult to identify with known DIB features since their spectral signature falls into the NIR region, which is difficult to explore from the astronomical point of view. Further 
astronomical surveys will be needed to improve the astronomical data in this range.

This comparative study has shown that most neutrals - with the possible exception of quaterrylene - can be definitely excluded as DIB carriers. It has also indicated that the less compact structures, such as diphenanthrofuran, decacyclene and 2, 2'-bis-triphenylyl, can also be excluded from future DIB searches. Several ionized PAHs (cations as well as anions) do however show possible correlations with DIBs. This is the case, for example, of the ions of terrylene, ovalene, hexabenzoperopyrene and dicoronylene. All are compact structures that are expected to be photostable in an extraterrestrial environment (Salama et al. 1996). The coincidences found in this work between the strong absorption bands of neutral quaterrylene, the terrylene ion, the hexabenzoperopyrene ion, and the dicoronylene ion and some DIBs make these specific species interesting candidates for future gas-phase studies.

In conclusion, given the large number of DIBs the probability of close matches between the laboratory and DIB spectra could be due to random chance. In particular since each new high resolution study of DIBs reveals a number of new weak features. We reiterate that a direct comparison of the MIS spectra of these large neutral and ionized PAHs with the spectra of a selected reddened star, HD183143, cannot yield to a decisive, unambiguous, identification of DIB carriers until gasphase laboratory data are available. This is due to shifts in band peak positions and to band profile broadening induced by the solid matrix in the spectrum of the isolated PAHs. Only gas phase measurements can provide access to intrinsic band positions and profiles that are necessary for the definitive identification of any specific molecular DIB carrier. A comparison between the MIS laboratory data and the astronomical observations is very useful, however, when matrix-to-gas-phase shifts are taken into account. The MIS data provide, then, an essential and unique guide for the selection of PAH ions to be studied under conditions that mimic closely the conditions reigning in the interstellar medium (i.e., in a free jet expansion).

The gas-phase, jet experiments which are just now developed (Biennier et al. 2002) will provide the much-needed data to definitively assess the validity of the PAH proposal with regards to the DIBs. More astronomical surveys of DIB objects are also needed, especially in the wavelength ranges that have not been well observed.

These laboratory experiments have been conducted in support of the experiment "ORGANIC" on the ISS (Ruiterkamp et al. 2001). This experiment will test the photostability of large carbon-bearing molecules during a long duration exposure in Earth orbit. Comparison of these laboratory spectra with the spectra of the post-flight samples will provide important information on the effects of space radiation on cosmic organic materials and will be reported separately.

Acknowledgements. These studies are part of the preparation for the ORGANICS experiment on the European EXPOSE facility on board the International Space Station (ISS). The authors acknowledge useful discussions with Lou Allamandola. RR acknowledges funding from SRON grant MG049. Additional support has been provided by NASA
(OSS Space Astrophysics Research and Analysis program) and ESA Space Science Dept.

\section{References}

Allamandola, L. J., Tielens, G. G. M., \& Barker, J. R. 1989, ApJS, 71,733

Bakes, E. L. O., \& Tielens, A. G. G. M. 1994, ApJ, 427, 822

Beals, G. H., \& Blanchet, G. H. 1937, PASP, 49, 224

Bettens, R. P. A., \& Herbst, E. 1996, ApJ, 468, 686

Biennier, L., Salama, F., Allamandola, L., Scherer, J., \& O’Keefe, A. 2002 , to be submitted

Beals, G. H., \& Blanchet, G. H. 1937, PASP, 49, 224

Bréchignac, P., \& Pino, T. 1999, A\&A, 343, L49

Cami, J., Sonnentrucker, P., Ehrenfreund, P., \& Foing, B. H. 1997, A\&A, 326, 822

Clar, E. 1964, Polycyclic Hydrocarbons (Academic Press, London)

Chen, B., \& Salama, F. 2001, J. Chem. Phys., submitted

Chlewicki, G., Groot, M. S., Van der Zwet, G. P., et al. 1987, A\&A, 173, 131

Douglas, A. E., \& Herzberg, G. 1941, ApJ, 94, 381

Ehrenfreund, P., D’Hendencourt, L., Verstraete, L., et al. 1992, A\&A, 259,257

Ehrenfreund, P., Foing, D’Hendecourt, L., Jenniskens, P., \& Desert, F. X. 1995, A\&A, 299, 213

Ehrenfreund, P., \& Foing, B. H. 1996, A\&A, 307, L25

Ehrenfreund, P., \& Charnley, S. B. 2000, ARA\&A, 38, 427483

Ehrenfreund, P., Sonnentrucker, P., O'Tuairisg, S., Cami, J., \& Foing, B. H. 2001, in Proceedings of the Workshop: The Bridge between the Big Bang and Biology, Stromboli 1999, Consiglio Nazionale delle Ricerche of Italy, ed. F. Giovannelli, Special Volume, 150

Ferlet, R., Roueff, E., Horani, M., \& Rostas, J. 1983, A\&A, 125, L5

Foing, B. H., \& Ehrenfreund, P. 1994, Nature, 369, 296

Foing, B. H., \& Ehrenfreund, P. 1997, A\&A, 317, L95

Freivogel, P., Fulara, J., \& Maier, J. P. 1994, ApJ, 431, L151

Galazutdinov, G. A., Musaev, F. A., Krelowski, J., \& Walker, G. A. H. 2000, Pubs. of the Astron. Soc. of the Pacific, 112, 648

Henning, Th., \& Salama, F. 1998, Science, 282, 2204

Herbig, G. H. 1967, in Radio Astronomy and the Galactic System, IAU Symp., 31, ed. H. van Woerden, 85

Herbig, G. H. 1975, ApJ, 196, 129

Herbig, G. H. 1988, ApJ, 331, 999

Herbig, G. H., \& Leka, K. D. 1991, ApJ, 382, 193

Herbig, G. H. 1993, ApJ, 407, 142

Herbig, G. H. 1995, ARA\&A, 33, 19

Herbst, E. 1995, Large Molecules in the Interstellar Medium, ASSL Vol. 202, The Diffuse Interstellar Bands, 307

Hudgins, D. M., \& Allamandola, L. J. 1999a, ApJ, 516, L41

Hudgins, D. M., \& Allamandola, L. J. 1999b, ApJ, 513, L69

Jacox, M. E. 1978, in Rev. Chem. Inter., ed. O. P. Strausz (Verlag Chemie International, Inc.: New York), 1

Jenniskens, P., \& Désert, X. 1993, A\&A, 274, 465

Jenniskens, P., \& Désert, X. 1994, A\&A, 106, 39

Joblin, C., Salama, F., \& Allamandola, L. 1999, J. Chem. Phys., 110, 7287

Krelowski, J., Sneden, C., \& Hiltgen, D. 1995, Plan. Space Sci., 43, 1195

Krelowski, J., Galazutdinov, G. A., \& Musaev, F. A. 1998, ApJ, 506, 926

Le Coupanec, P., Rouan, D., Moutou, C., \& Léger, A. 1999, A\&A, 347, 669

Lucas, R., \& Liszt, H. S. 1997, Molecules in Astrophysics: Probes and Processes, ed. E. F. van Dishoeck (Dordrecht: Kluwer), 421

McKelar, A. 1940, Publ. Astr. Soc. Pacific, 52, 187 
Mennella, V., Colangeli, L., Brucato, J. R., et al. 1999, Adv. Space Res., 24, 439

Merril, P. W. 1934, PASP, 46, 206

Puget, J. L., \& Leger, A. 1989, ARA\&A, 27, 161

Romanini, D., Biennier, L., Salama, F., et al. 1999, Chem. Phys. Lett., 303,165

Ruffle, D. P., Bettens, R. P. A., Terzieva, R., \& Herbst, E. 1999, ApJ, 523,678

Ruiterkamp, R., Ehrenfreund, P., Foing, B., \& Salama, F. 2001, Conf. Proc. First European Workshop Exo-/Astro-Biology SP-496, ed. P. Ehrenfreund, O. Angerer, \& B. Battrick (ESA), 137

Salama, F., \& Allamandola, L. J. 1991 J. Chem. Phys., 94, 6964

Salama, F. 1996, Low Temperature Molecular Spectroscopy, 483, ed. R. Fausto (Kluwer), 169

Salama, F., Bakes, E. L. O., Allamandola, L. J., \& Tielens, A. G. G. M. 1996, ApJ, 458, 621

Salama, F. 1999, Solid Interstellar Matter: The ISO Revolution 458, ed. L. d'Hendecourt, C. Joblin, \& A. Jones (EDP Sciences, Springer-Verlag, Les Ulis), 65

Salama, F., Galazutdinov, G. A., Krełowski, J., Allamandola, L. J., \& Musaev, F. A. 1999, ApJ, 526, 265
Sanner, F., Snell, R., \& Vanden Bout, P. 1978, ApJ, 226, 460

Sarre, P. J., Miles, J. R., \& Scarrott, S. M. 1995, Science, 269, 674

Sonnentrucker, P., Cami, J., Ehrenfreund, P., \& Foing, B. H. 1997, A\&A, 327, 1215

Swings, P., \& Rosenfeld, L. 1937, ApJ, 86, 483

Tielens, A. G. G. M., \& Snow, T. P. (eds.) 1995, The Diffuse Interstellar Bands, IAU Coll. 137 (Kluwer, Dordrecht)

Tielens, A. G. G. M., Hony, S., van Kerckhoven, C., \& Peeters, E. 1999, ESA SP-427, The Universe as Seen by ISO, 427, 579

Torres-Godgen, A. V. 1990, PASP, 102, 1406

Ó Tuairisg, S., Cami, J., Foing, B. H., Sonnentrucker, P., \& Ehrenfreund, P. 2000, A\&A, 142, 225

Tulej, M., Kirkwood, D. A., Pachkov, M., \& Maier, J. P. 1998, ApJ, 506, L69

Vuong, M. H., \& Foing, B. H. 2000, A\&A, 363, L5-L8

Vuong, M. H., \& Foing, B. H. 2002, in preparation

Walker, G. A. H., Webster, A. S., Bohlender, D. A., \& Krełowski, J. 2001, ApJ, 561, 272

Wilson, R. 1958, ApJ, 128, 57 